Draft VERSiOn SEPTEMBER 17, 2018

Preprint typeset using $\mathrm{LAT}_{\mathrm{E}} \mathrm{X}$ style emulateapj v. 11/10/09

\title{
SUBLUMINOUS TYPE IA SUPERNOVAE AT HIGH REDSHIFT FROM THE SUPERNOVA LEGACY SURVEY ${ }^{1}$
}

\author{
S. González-Gaitán ${ }^{2}$, K. Perrett ${ }^{2,3}$, M. Sullivan ${ }^{4}$, A. Conley $^{2,5}$, D. A. Howell ${ }^{6,7}$, R. G. Carlberg $^{2}$, P. Astier $^{8}$, \\ D. BAlam ${ }^{9}$, C. Balland ${ }^{8}$, S. BAsa ${ }^{10}$, D. Fouchez ${ }^{11}$, J. GuY ${ }^{8}$, D. Hardin ${ }^{8}$, I. M. Hook. ${ }^{9,12}$, R. PAin ${ }^{8}$, \\ C. J. Pritchet ${ }^{13}$, N. Regnault ${ }^{8}$, And J. Rich ${ }^{14}$ \\ ${ }^{1}$ Based on observations obtained with MegaPrime/MegaCam, a joint project of CFHT and CEA/DAPNIA, at the \\ Canada-France-Hawaii (CFHT) which is operated by the National Research Council (NRC) of Canada, the Institut National des Sciences \\ de l'Univers of the Centre National de la Recherche Scientifique (CNRS) of France, and the University of Hawaii. This work is based in \\ part on data products at the Canadian Astronomy Data Centre as part of the Canada-France-Hawaii Telescope Legacy Survey, a \\ collaborative project of NRC and CNRS. \\ ${ }^{2}$ Department of Astronomy and Astrophysics, University of Toronto, 50 St. George Street, Toronto, ON, M5S 3H4, Canada \\ ${ }^{3}$ Network Information Operations, DRDC Ottawa, 3701 Carling Avenue, Ottawa, ON, K1A 0Z4, Canada \\ ${ }^{4}$ Department of Physics (Astrophysics), University of Oxford, DWB, Keble Road, Oxford, OX1 3RH, UK \\ ${ }^{5}$ Center for Astrophysics and Space Astronomy, University of Colorado, 593 UCB, Boulder, CO, 80309-0593, USA \\ ${ }^{6}$ Las Cumbres Observatory Global Telescope Network, 6740 Cortona Dr., Suite 102, Goleta, CA 93117, USA \\ ${ }^{7}$ Department of Physics, University of California, Santa Barbara, Broida Hall, Mail Code 9530, Santa Barbara, CA 93106-9530, USA \\ ${ }^{8}$ LPNHE, Université Pierre et Marie Curie Paris 6, Université Paris Diderot Paris 7, CNRS-IN2P3, 4 Place Jussieu, 75252 Paris Cedex \\ 05, France \\ ${ }^{9}$ Dominion Astrophysical Observatory, Herzberg Institute of Astrophysics, 5071 West Saanich Road, Victoria, BC, V9E 2E7, Canada \\ ${ }^{10}$ Laboratoire d'Astrophysique de Marseille, Pôle de l'Étoile Site de Château-Gombert, 38, rue Frédéric Joliot-Curie, 13388 Marseille \\ cedex 13, France \\ ${ }^{11}$ CPPM, CNRS-IN2P3 and University Aix Marseille II, Case 907, 13288 Marseille cedex 9, France \\ ${ }^{12} \mathrm{INAF}$, Osservatorio Astronomico di Roma, via Frascati 33, 00040 Monteporzio (RM), Italy \\ ${ }^{13}$ Department of Physics \& Astronomy, University of Victoria, PO Box 3055, Stn CSC, Victoria, BC, V8W 3P6, Canada and \\ ${ }^{14}$ DSM/IRFU/SPP, CEA-Saclay, F-91191 Gif-sur-Yvette, France \\ Draft version September 17, 2018
}

\begin{abstract}
The rate evolution of subluminous Type Ia Supernovae is presented using data from the Supernova Legacy Survey. This sub-sample represents the faint and rapidly-declining light-curves of the observed supernova Ia (SN Ia) population here defined by low stretch values $(s \leq 0.8)$. Up to redshift $z=0.6$, we find 18 photometrically-identified subluminous SNe Ia, of which six have spectroscopic redshift (and three are spectroscopically-confirmed SNe Ia). The evolution of the subluminous volumetric rate is constant or slightly decreasing with redshift, in contrast to the increasing SN Ia rate found for the normal stretch population, although a rising behaviour is not conclusively ruled out. The subluminous sample is mainly found in early-type galaxies with little or no star formation, so that the rate evolution is consistent with a galactic mass dependent behavior: $r(z)=A \times M_{g}$, with $A=(1.1 \pm 0.3) \times 10^{-14} \mathrm{SNe} \mathrm{yr}^{-1} M_{\odot}^{-1}$.

Subject headings: supernovae: general
\end{abstract}

\section{INTRODUCTION}

While it is commonly agreed that Type Ia Supernovae (SNe Ia) are thermonuclear disruptions of mass accreting C-O white dwarfs (WDs) in a binary system (Hovle \& Fowler 1960), the physics of the explosion and the nature of the companion are still under discussion. The study of the properties and environments of SNe Ia can help us solve the progenitor question. Successful progenitor and explosion models need to explain the variety in light-curve shape and spectra of SNe Ia. Recent multidimensional simulations of the explosion Gamezo et al. 2005; Livne et al. 2005; Kuhlen et al. 2006; Kasen et al. 2009) show the asymmetric character of the delayed detonation and succeed to explain the scatter in the widthluminosity relation of the normal SNe Ia, although complications for the extreme SNe Ia still exist. In particular, subluminous SNe Ia - a group of objects considerably fainter at peak (up to $2 \mathrm{mag}$ ), with faster light-curves, redder at early phases, with distinct spectral characteristics such as Ti II and enhanced Si II - pose a challenge to any successful progenitor and explosion theory.
The prototypical example of a subluminous $\mathrm{SN}$ Ia is SN1991bg (e.g. Filippenko et al. 1992) but many other examples have been discovered and studies of their properties relative to the bulk sample have been undertaken (Garnavich et al. 2004; Taubenberger et al. 2008; Kasliwal et al. 2008; Hicken et al. 2009). Subluminous SNe Ia are predominantly found in galaxies with older stellar populations, such as ellipticals and early-type spirals (Howell 2001), in contrast to the preference for the late-type, star forming hosts favoured by bright, slow declining SNe Ia (Hamuy et al. 1996, 2000). Subluminous SNe Ia also occur exclusively in massive galaxies whereas the normal sample spans a wider host stellar mass range (Neill et al. 2009). These observations set constraints on the delay-time - the time between the formation of the binary system and subsequent SN explosion - ranging from $<1$ Gyr for SNe occurring in star-forming regions to several Gyrs for SNe in quiescent environments. They could as well hint at differences in the metallicity abundance of the progenitors, due to the mass-metallicity relation (Tremonti et al. 2004), i. e., subluminous SNe Ia happen in more metal-rich environments.

Based on a sample from the Lick Observatory Super- 
nova Search (LOSS) and the Beijing Astronomical Observatory Supernova Survey (BAOSS), Li et al. (2001, 2010a) found that $17.9_{-6.2}^{+7.2} \%$ of all local SNe Ia are $1991 \mathrm{bg}$-like, a possible overestimation as these surveys were host-targeted and could have systematically sampled brighter and more massive galaxies. The current observed subluminous sample remains a small fraction of the overall SN Ia population, although new and recent surveys are actively looking for them (Hicken et al. 2009). At high redshift, they are challenging to identify spectroscopically, and the current high- $z$ SN surveys usually preferentially target normal SNe Ia for cosmological purposes. As a result, no $91 \mathrm{bg}$-like $\mathrm{SNe}$ have been located at $z>0.1$. For example, both, the Supernova Legacy Survey (SNLS) and the ESSENCE survey, report no spectra of $91 \mathrm{bg}$-like objects at $z>0.1$, although this is consistent with their selection effects (Bronder et al. 2008; Folev et al. 2009).

This raises an obvious question: is this non detection simply due to the difficulty of detecting and classifying these objects, or is the relative frequency actually lower at high redshift because there has not been enough time for them to explode as SNe Ia (Howell 2001)? Problems in the detection and classification arise from their intrinsic faintness, their rapidly evolving light-curves (which cause them to spend less time above the detection threshold for the same brightness), and their tendency to occur in brighter galaxies where the low contrast between the SN and host makes spectroscopy difficult. In this paper, we look for the fastest (and therefore faintest) SNe Ia at $z>0.1$ in the Supernova Legacy Survey (SNLS) (Astier et al. 2006). Even without spectroscopic followup, we can use the excellent multi-band light-curves of the SNLS to look for subluminous SN Ia candidates by fitting subluminous SN Ia LC templates to the photometric data.

Beyond a simple detection, even more enlightening would be a measurement of the evolution in the volumetric rate of these objects. As it is a convolution of the starformation history and the delay-time distribution, the SN Ia rate, in particular for sub-samples of SNe Ia, can test different progenitor scenarios. Rates for SNe Ia have been measured by several groups (e.g. Pain et al. 1996, 2002; Cappellaro et al. 1999; Dahlen et al. 2004, 2008; Neill et al. 2006; Dildav et al. 2008, 2010; Perrett et al. 2010a; Li et al. 2010b). Combining the results one finds an increase with redshift up to $z \simeq 1.0$, although with large spread among surveys. If the rate evolution for the individual SN Ia populations differ, their delay-times must consequently vary and imply different conditions for the progenitors. As we probe higher redshifts, the SN Ia environments will differ from the local sample, and will be reflected in the rates. If the fraction of high-mass (and high-metallicity) hosts was lower in the past, we would expect a similar behaviour for the rate of subluminous SNe Ia.

By constraining the delay-time distribution, we seek to improve our understanding of the progenitors of subluminous SNe Ia. One can attempt to explain their progenitors in the SN Ia progenitor framework, where two main scenarios have been envisaged: the single-degenerate (SD) model, in which a non-degenerate companion donates $\mathrm{H} / \mathrm{He}$-rich material to a WD near the Chan- drasekhar mass (Whelan \& Iben 1973; Nomoto et al. 1984), and the double-degenerate scenario of two WDs coalescing (Iben \& Tutukov 1984; Webbink 1984). In the SD scenario the delay-time is set by the age of the donor while in the DD model it depends on the age of the secondary and the merging time of the two WDs through gravitational wave radiation. The merging of two WDs has recently shown to be a viable mechanism, under certain conditions, for a successful subluminous explosion (Pakmor et al. 2010).

There is also a variety of independent mechanisms for subluminous explosions that explain the faintness by requiring a smaller burned mass of ${ }^{56} \mathrm{Ni}$. Instead of a common explosion model for the whole range of SNe Ia, the delayed detonation (Mazzali et al.|2007) and other mechanisms like pure deflagrations or sub-Chandrasekhar $\mathrm{CO}$ or $\mathrm{O}-\mathrm{Ne}-\mathrm{Mg}$ WD off-center detonations (e.g. Livne 1990; Wooslev \& Weaver 1994; Filippenko et al. 1992; Isern et al. 1991) provide less luminosity although with some observational discrepancies (Hoeflich \& Khokhlov 1996). A failed neutron star model, in which $\mathrm{C}-\mathrm{O}$ is accreted rapidly and ignited on the surface of the WD also leads to a faint SN Ia (Nomoto \& Iben 1985). Recently proposed models of WD collisions in dense environments could potentially lead to subluminous explosions (Raskin et al. 2009a; Rosswog et al. 2009). Finally, a theoretically-motivated group of unusual objects, the so called SNe .Ia ("dot Ia") introduced by Bildsten et al. (2007), are the final helium shell flashes of AM CVn binaries. These are too dim and evolving too rapidly to be classical subluminous SNe Ia, but possible candidates are starting to appear (Poznanski et al. 2010; Kasliwal et al. 2010).

With a detection of subluminous SNe Ia at $z>0.1$, the measurement of the rate evolution and study of their host environments, we can start setting constraints on the evolution of these objects and their properties, their delay-times and progenitors. This paper is a companion to Perrett et al. (2010a), who have carried out a rate study of normal SN Ia sub-samples using the same SNLS data sample. We complement that study for the faint and short-lived SN Ia population up to $z=0.6$. We use "stretch" as a LC-shape parameter (Perlmutter et al. 1997; Goldhaber et al. 2001; Conley et al. 2008) and define hereafter subluminous objects as SNe Ia with $s \leq 0.8$, inspired by the behavior of the color-stretch and magnitude-stretch relations (\$2.1). This value is higher than the ones inferred from other studies (e.g. Taubenberger et al. 2008; Li et al. 2010a), as will be shown later. We emphasize therefore that our sample is less extreme and call it hereafter "low-stretch" (low-s) sample.

A brief outline of the paper follows. In $\$ 2$ we investigate the nearby training sample of low- $s$ SNe Ia and find the necessary LC relations for creating a template. 33 highlights the observations and candidate selection of the SNLS. With this, we measure the rate as a function of redshift in 4 taking into account systematic effects from possible errors in the estimated LC parameters and contamination from core-collapse supernovae (CC-SNe). The discussion of the rate measurements together with the investigation of the host galaxy properties of the candidates follow in $\$ 5$ and we conclude in $\$ 6$. We use a flat cosmology with $\left(\Omega_{m}, \Omega_{\Lambda}\right)=(0.27,0.73)$ and $h=0.7$. 


\section{LOCAL RELATIONS FOR LOW-STRETCH SNE IA}

As shown by Garnavich et al. (2004); Taubenberger et al. (2008); Kasliwal et al. (2008) and Hicken et al. (2009), subluminous SNe Ia have clear photometric characteristics: red colors, a faint peak brightness, and a steeper relationship between LC shape and peak magnitude. In this section we derive new parameter relations based on a training sample of subluminous objects at low redshift. These relations will be of great importance to define proper low- $s$ SN Ia LC templates and to correctly calculate the SNLS survey efficiency.

We use a local training sample from the literature (the low- $z$ Constitution set and low- $s$, Table 10 that spans the stretch range, $0.45<s<1.30$, so that the behavior of low- $s$ SNe can be compared with the normal population. Adequate light-curve phase coverage is ensured by requiring observations in at least two filters between -15 and 8 and between 5 and 25 effective days (corrected for stretch and redshift) after maximum brightness in $B$-band. We also include a sample at higher redshift from the SNLS consisting of spectroscopically-confirmed $\mathrm{SNe}$ Ia over $0.3<z<0.6$, which provides wider wavelength coverage compared to the low- $z$ sample.

We perform SN Ia light curve fits to the entire sample with a modified version of the SiFTO algorithm (Conley et al. 2008). SiFTO manipulates spectral energy distribution (SED) templates to model SN Ia LCs. It has a wavelength-stretch dependence derived from strictly normal SNe Ia, which was removed for the modified version used here. Although SiFTO was built with a training sample that excluded peculiar objects like SN1991bg, we obtain very good fits to subluminous data down to $s \simeq 0.65$ and reasonable fits to lower stretch $\mathrm{SNe}$ (see Figure 11). Surprisingly, this template performs better than those built purely from subluminous objects like SN1991bg, which may have extreme features not suitable for all $s \leq 0.8$ objects. The success of the fitting is partly because SiFTO fits each band independently without imposing any color-stretch relation, and so the unusual colors of subluminous SNe Ia do not pose a problem. The algorithm provides the LC parameters such as stretch and peak magnitudes to get colors at maximum $B$-band brightness with their respective errors, as well as quality-of-fit parameters.

We use a reduced overall goodness-of-fit $\chi_{\nu}^{2}<5$ cut for normal and a higher $\chi_{\nu}^{2}<11$ for low- $s$ objects to account for the poorer fits of the more extreme objects. The final training set consists of: 207 low- $z$ SNe Ia, of which 44 are low- $s$ (see Table 1) with $s$ down to $s=0.45$, and 106 SNLS spectroscopic SNe Ia of which 3 are low-s. A list of all culls is shown in Table 3. We note that not all of the low-s SNe Ia listed by Neill et al. (2009) (Table 1) pass our selection criteria. Also, their stretch values for low- $s$ objects are in average lower than ours. This is due to the modified version of SiFTO we use, which assumes a common single stretch independent of wavelength and which has been more extensively tested for low- $s$ SNe Ia. Additionally, despite our light curve coverage criteria, one low- $s$ candidate (SN2001da) does not have many data points and results in very different stretch $(s=0.89)$ when fit through the method of Neill et al. (2009). We take this supernova out of our analysis.
In order to develop tools to photometrically identify low- $s$ SNe Ia and distinguish them from other transients, we also include a nearby and a high- $z$ SNLS sample of spectroscopically-identified non-type Ia supernovae. 14 nearby and 21 SNLS CC-SNe are properly fit to a SN Ia SiFTO template, and 10 of those are assigned a $s \leq 0.8$ stretch (6 of which are low- $z$, see Table 2). This set of contaminants will give us an idea of the systematic uncertainties (more in 4.3 ).

Our limiting definition of low- $s$ objects at $s=0.8$ is inspired by the color-stretch and magnitude-color-stretch relations and is different from the "subluminous" definition studied in other surveys. We can compare it to the SALT2 LC-fitter (Guv et al. 2007) using similar equations to Eq. 6 in Guy et al. (2010), obtaining that $s=0.8$ for our modified SiFTO version corresponds to $X_{1} \simeq-1.85$. By directly matching subluminous $\mathrm{SNe}$ of Taubenberger et al. (2008) and fitting a linear relation between their $\Delta m_{15}(B)$ parameter (the amount of $B$-band magnitudes the SN has declined 15 days after maximum) and our SiFTO stretch, we obtain a corresponding value of $\Delta m_{15}(B) \simeq 1.54$. This value is lower than the one they find to separate their sample $(\simeq 1.70$, which would correspond to $s \simeq 0.72$ ) and confirms again that our sample is not as extreme.
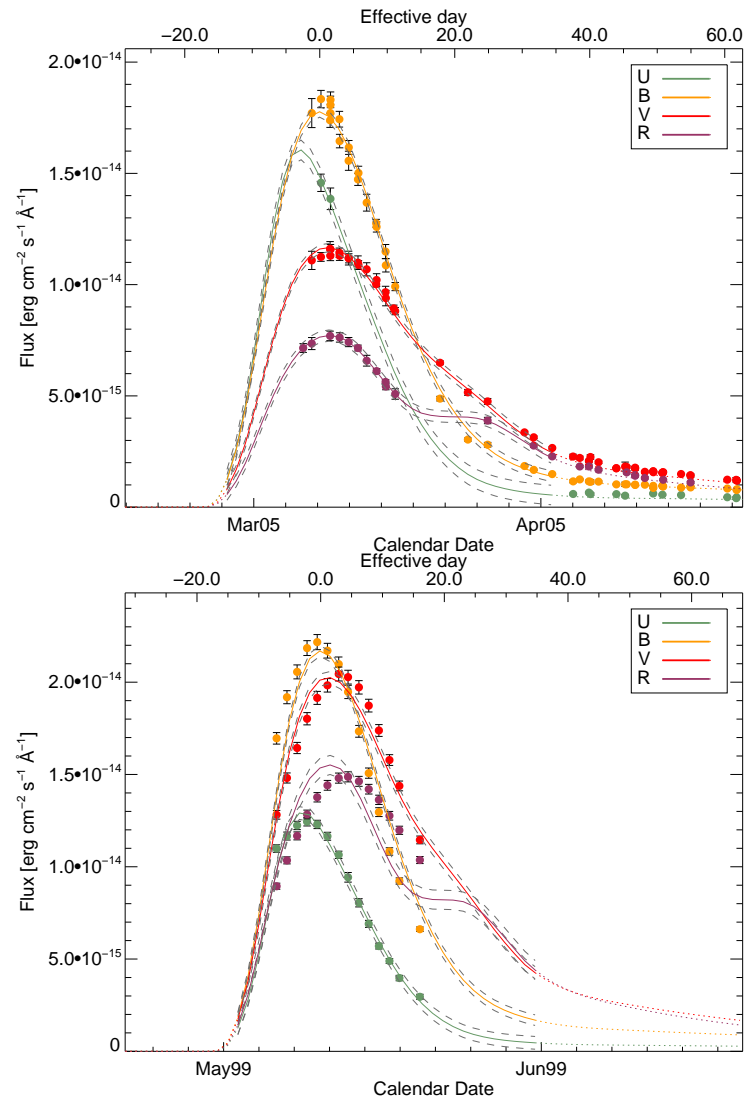

Fig. 1. - Example SiFTO LC fits of low-s SN2005am (Hicken et al. 2009; Li et al. 2006) with stretch 0.71 and overall $\chi_{\nu}^{2}=0.6$ and SN1999by (lower) with stretch 0.61 and $\chi_{\nu}^{2}=8.5$. The fit is done for all points between -15 and 35 days in restframe.

\subsection{Color-stretch relations}


Modeling the colors of SNe Ia is fundamental for the LC fit and candidate selection of 93 , as well as for the study of the recovery efficiency needed for the rate calculation of 84 . The challenge in modeling SN Ia colors resides in the difficulty of disentangling intrinsic and extrinsic colors, the latter coming mainly from reddening of the host galaxy. As explained in Conley et al. (2008), the SiFTO method does not impose a single color model across all filters, which would probably not encompass the whole range of SN Ia variability; rather the SED is adjusted to match the observed colors in each filter.

We first remove highly reddened objects by the foreground Milky Way using the Schlegel et al. (1998) dust maps and a cut of $E(B-V)_{\mathrm{MW}}<0.15$. To get an idea of the intrinsic color-stretch relation of the objects, we take the transients that are hosted in less extincted passive galaxies, i.e. in galaxies catalogued as E/S0 for the nearby sample from the NED database 1 and in galaxies with no star formation for the SNLS (Sullivan et al. 2006b). A color similar to $B-V$ at B-band maximum brightness, $c$, as a function of stretch for these objects is shown in Figure 2 (filled circles and squares).

We create a "low-extinction sample" by taking all SNe, regardless of their hosts, with a color below the upper limit from the maximum found for the SNe in only quiescent environments, $c<0.37$. This is only done for normal- $s$ SNe Ia, i. e. $s>0.8$, which have a relatively flat color-stretch relation. Low-stretch SNe Ia become redder at a steeper rate with decreasing stretch and are hosted in less dusty environments, so we do not use such a maximum color cut, although we remove known extremely extincted objects (e.g. SN1986G, SN2006br and SN2007ax). The fit to this larger sample (open and filled circles and squares) in Figure 2 is well represented by a piecewise linear model with a break at $s=0.77$ (solid line):

$$
c(s)= \begin{cases}C_{0}+\gamma_{1}(s-1) & (s>0.77) \\ C_{0}+\gamma_{2}(s-1)+C_{\text {match }} & (s \leq 0.77)\end{cases}
$$

The fit parameters are $C_{0}=0.08 \pm 0.02, \gamma_{1}=-0.06 \pm$ $0.02, \gamma_{2}=-2.79 \pm 0.07$ and the transition stretch value $s=0.77 \pm 0.01 . C_{\text {match }}=-0.23\left(\gamma_{1}-\gamma_{2}\right)$ is a correction factor to unite both lines at $s=0.77$. The transition value for stretch $(s=0.77)$, together with the one found for the magnitude-color-stretch relation in next section $(s=0.82)$, argues for our chosen limiting value of $s \simeq 0.8$ between low- $s$ and normal- $s$. The piecewise linear model has the best fit (reduced $\chi_{\nu}^{2}$ of 23.4) among other models (e. g. exponential with $\chi_{\nu}^{2}=25.0$ or power law with $\left.\chi_{\nu}^{2}=25.9\right)$. The figure shows that low-stretch SNe Ia are not only redder but their color-stretch relation is much steeper than for normal SNe Ia. Errors in stretch and color were propagated in the fits. Allowing a fit to all objects, also extincted ones, provides consistent results (with a transition stretch at $s=0.83$ ) and is also shown in Figure 2. It is worth to mention that if we force a piecewise linear model with a fixed lower transition value of $s=0.7$, more similar to characteristic "sub-

1 The NASA/IPAC Extragalactic Database (NED) is operated by the Jet Propulsion Laboratory, California Institute of Technology, under contract with the National Aeronautics and Space Administration luminous" surveys (Taubenberger et al. 2008), the fit is slightly worse $\left(\chi_{\nu}^{2}=24.6\right)$ and the slopes steeper. Due to the large scatter of colors at low- $s$, a range of consistent models (with $\chi^{2}<\chi_{\min }^{2}+2$ ) is obtained for transition values of $0.69-0.87$.

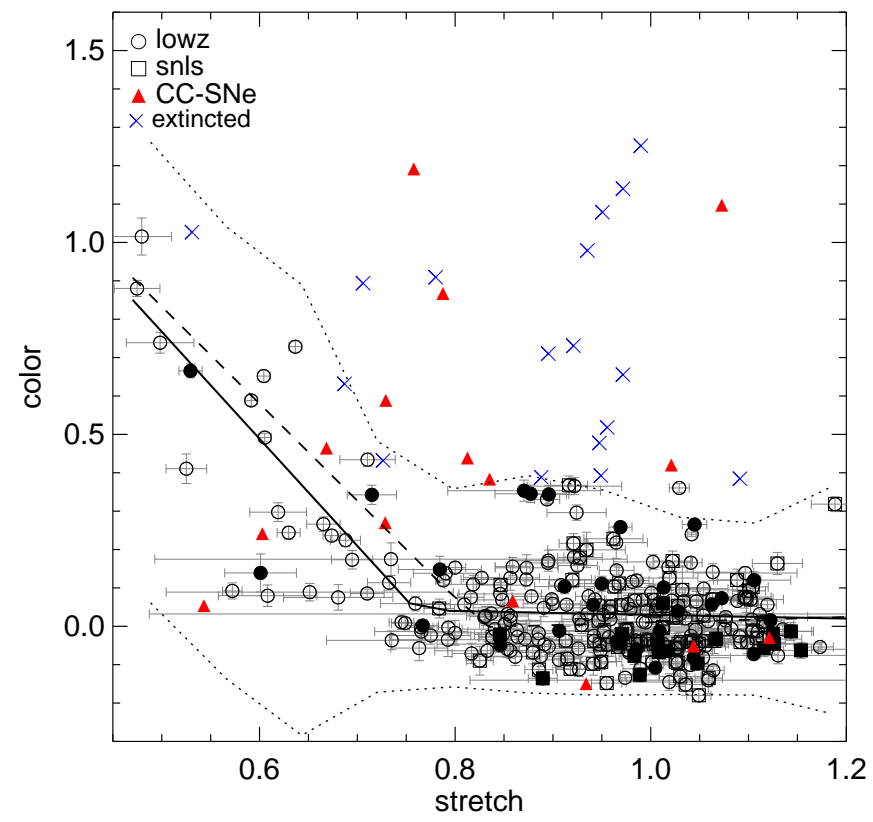

FIG. 2.- Color as a function of stretch for the local SN Ia sample (circles) and $0.3<z<0.6$ SNLS sample (squares). The filled points represent $\mathrm{SNe}$ in passive galaxies and the fit to exclusively those objects is the dashed line, a two-piece linear model changing at $s \simeq 0.8$. The "low-extinction" sample (open and filled black points) represent all objects with $c<0.37$ regardless of the host galaxy. The solid line is the best two-piece linear fit to this sample and the dotted lines are the the $2 \times 2 \sigma$ error-snakes. High-extinction $\mathrm{SNe}$ (blue crosses) are above the maximum possible intrinsic colors calculated from the $\mathrm{SNe}$ in passive galaxies and were not included in the fits. CC-SNe (red triangles) are also shown.

\subsection{Luminosity-color-stretch}

We next derive a relation between the absolute peak magnitude as a function of stretch and color. To limit the effects of peculiar velocities, we require SNe in our training sample to have either $z_{\mathrm{CMB}}>0.01$ (CMB frame redshift) or a known distance to the host taken from the NED database.

We fit simultaneously magnitude, stretch and color to different models. The best one is again bilinear in both, stretch and color. The absolute magnitude shows a steeper slope as a function of color for redder objects changing at $c \simeq 0.07$ (upper panel, Figure 31). Against stretch, the magnitude values (lower panel, Figure 3) are also well fit by a bilinear model with a shallower slope for the low-s stretch range. The effects in the differing slopes are not as strong as for the color-stretch relation but they will make the posterior analysis more precise. The errors in the absolute magnitude (from the apparent magnitude and redshift errors) and in the stretch and color are included in the fit. The magnitude-stretch-color relation can be summarized as follows:

$$
M_{B}=M+\alpha_{[1,2]}(s-1)+\beta_{[a, b]} c+M_{[2, b]}^{m a t c h}
$$


with $M=-19.11 \pm 0.01$, and where $\alpha_{1}=-1.19 \pm 0.03$ and $\alpha_{2}=-0.54 \pm 0.09$ are respectively used for stretch values greater and lower than the transition value of $s=$ $0.82 \pm 0.02$. $\beta_{a}=3.00 \pm 0.02$ and $\beta_{b}=2.15 \pm 0.05$ are used for values of $c$ greater and lower than $0.07 \pm 0.01$, respectively. $M_{2}^{\text {match }}=-0.18\left(\alpha_{1}-\alpha_{2}\right)$ and $M_{b}^{\text {match }}=$ $0.07\left(\beta_{a}-\beta_{b}\right)$ unite the fit at the changing values. This model results in a slightly better fit $\left(\chi_{\nu}^{2}=19.6\right)$ than other models (e. g. exponential -in stretch and colorwith $\left.\chi_{\nu}^{2}=19.9\right)$.


FIG. 3.- Absolute magnitude (corrected for stretch from Eq. 2 i. e. $\left.M_{B}^{s-\text { corr }}=M_{B}-\alpha_{[1,2]}(s-1)-M_{2}^{\text {match }}\right)$ vs color (upper $)$ and absolute magnitude (corrected for color, i. e. $M_{B}^{c-\text { corr }}=M_{B}-$ $\beta_{[a, b]} c-M_{b}^{\text {match }}$ ) vs stretch (lower) for the training sample. The solid lines represent the best fit model, a two-piece linear model for each case. The dashed lines are respectively the $2 \times 2 \sigma$ error-snakes. CC-SNe (red triangles) are also shown.

We include an asymmetric error-snake on these relations, shown by the dotted lines in Figures 2 and 3 . These delimit (twice) the region within which $95 \%$ of all SNe Ia in each bin of 0.1 in color and 0.07 in stretch are found around the best model. The width of the re- gion has been doubled to allow for the larger photometric scatter of the high- $z \mathrm{SNe}$, which will be important later in $\$ 3.2$. This region also covers any uncertainties in the relations modeled in this section and allows for scatter in the color of the SNe. Requiring all objects to be inside those error-snakes, i.e. color-stretch, magnitudecolor and magnitude-stretch, we find that $\simeq 90 \%$ of the normal and low- $s$ SNe Ia pass the cut (see Table 3). However, only 2 contaminants (all SNe Ib/c) survive the same cull, $\mathrm{a} \simeq 6 \%$ of the total of contaminants. We find this tradeoff to be beneficial. Reducing the contamination is useful when limiting the systematic errors as discussed in 4.3 .

\section{HIGH- $Z$ LOW-STRETCH CANDIDATE SELECTION}

The search component of the SNLS, carried out using the Canada-France-Hawaii Telescope (CFHT), is a wide field imaging survey well-suited to the detection of SNe Ia in the redshift range $0.1 \leq z \lesssim 1.0$ (Astier et al. 2006). It is a rolling search where four $1^{\circ} \times 1^{\circ}$ fields (D1 to D4) are imaged every few days, so that a well sampled LC is obtained in four filters $\left(g_{M} r_{M} i_{M} z_{M}\right)$ for each discovered supernova. Good candidates are followed-up spectroscopically. Because they are faint, less frequent, and not vital for cosmological studies, typical subluminous $\mathrm{SNe}$ Ia are not prioritized in the spectroscopic followup (Sullivan et al. 2006a; Perrett et al. 2010b). We can still use the well-sampled SNLS multi-wavelength LCs to photometrically identify these objects. The method used resembles the one for the normal-stretch population of Perrett et al. (2010a, hereafter P10), although with appropriate adjustments for the low- $s$ objects. This makes a direct comparison with the different stretch populations possible.

During the first 4 years of operation, up to July 2007, the candidate database consists of more than 5000 photometric transients including AGN, non-SNe and CC-SNe. As in P10, candidates in masked areas of the deep stacks are removed, as well as objects that lack the necessary LC coverage for a good photometric identification and parameterization. These observational criteria reduce the sample to half and are the same ones used for the SNLS training sample in 92 at least one $g_{M}$ observation between -15 and 5 days of maximum, at least one $r_{M}$ and one $i_{M}$ observations between -15 and +2.5 days of maximum, at least one $r_{M}$ and one $i_{M}$ observations between -9 and +7 days of maximum, and at least one $r_{M}$ or one $i_{M}$ observation between +5 and +20 days of maximum.

\subsection{Redshift estimation and LC fit}

In $\mathrm{P} 10$, accurate photometric redshifts from the $\mathrm{SNe}$ were obtained with the use of two normal SN Ia LC fitters. Their approach requires a good spectroscopic sample for testing the method and the precision of the final photometric redshifts. We do not possess any $91 \mathrm{bg}$-like SN Ia spectrum at high redshift with clear Ti II features, only 3 low- $s$ objects at the border of our defintion, $s \lesssim 0.8$, that have spectra of rather normal SNe Ia. The high degree of degeneracy in the LC fits, particularly with stretch and redshift, which compete to make an object appear faint, demand an unbiased redshift estimate that will lead to appropriate LC parameters like stretch and color. 
Several hundred objects of the 4-year dataset possess spectroscopic redshifts (spec-z) from either the SN or from the host galaxy. For the SN-derived redshifts, the SN type has been determined from the spectra in the majority of cases (Howell et al. 2005; Bronder et al. 2008: Ellis et al. 2008; Balland et al. 2009). Most of these are normal SNe Ia (413), although a good sample of CC objects is also available (78). The host spec- $z$ include data from DEEP/DEEP2 (Davis et al. 2003), VVDS (Le Fèvre et al. 2005), zCOSMOS (Lilly et al. 2007) and VIMOS (Jönsson et al. 2010). Many candidates without spectroscopic redshifts have host photometric redshifts from the SNLS broad-band photometry either using the spectral connectivity analysis method of Freeman et al. (2009), or, when no match is found, through fits to spectral energy distributions as in Sullivan et al. (2006b). The resulting host photo- $z, z_{\text {hostphot }}$, are very good to $z \simeq 0.75$ with $\frac{|\Delta z|}{\left(1+z_{\text {spec }}\right)}=0.042$ and a low $(\sim 5 \%)$ catastrophic failure rate $\left(\frac{\left|z_{\text {spec }}-z_{\text {hostphot }}\right|}{\left(1+z_{\text {spec }}\right)}>0.15\right)$.

Fortunately, some of those SNe Ia with catastrophic redshifts can be identified with the help of the two LC fitters of P10 adjusted for low-s SN: estimate_sn (Sullivan et al. 2006a) and the aforementioned modified version of SiFTO (see \$2). Their use in the multi-step process explained below provides good quality-of-fit parameters capable of distinguishing $\mathrm{SNe}$ Ia from CC-SNe, but also offer an independent mechanism for obtaining photometric redshifts using the $\mathrm{SN}, z_{\mathrm{SNphot}}$, in cases where host redshifts are lacking.

Once the redshift is known, SiFTO is used to obtain the stretch and color of each SN. Tests on the local sample (\$2) show that it works well without imposing any stretch-color model. As shown in Figure 2 of P10, the derived stretch is largely independent of the redshift because the rise and fall of SNe Ia are faster at shorter wavelengths, which largely compensates for redshift uncertainties. In Figure 4, we demonstrate that this behavior extends to low- $s$ objects. Shifting the redshift - either photometric or spectroscopic- for each $\mathrm{SN}$ in our final low- $s$ sample (see 33.2 ) by as much as $\Delta z= \pm 0.3$, we obtain a stretch variation lower than $\sim s \pm 0.1$. While this shows more dependence on redshift than the normalstretch population, it still allows us to obtain accurate stretch estimates even in the presence of moderate redshift uncertainties.

The estimate_sn routine is described in more detail in Sullivan et al. (2006a). It performs a grid search on the $\chi^{2}$ of a fit to SN photometry in flux space to a fiveparameter model, including redshift. For this analysis, we modified it to include low- $s$ objects using the colorstretch relations of Eq. 1, and the magnitude relation of Eq. 2. This fit depends on the assumed values of the cosmological parameters.

We calculated the SN photo- $z$ for all objects in a similar fashion to P10 using the following multi-step process: First, we use estimate_sn to get a preliminary redshift estimate. We then use this redshift as a fixed input to a SiFTO fit to obtain an improved stretch measurement. Finally, we feed back this stretch to a final estimate_sn fit as a fixed parameter to obtain a more accurate $z_{\mathrm{SNphot}}$. We use this SN photo- $z$ for SNe that do not have any other redshift (spectroscopic or host photometric).
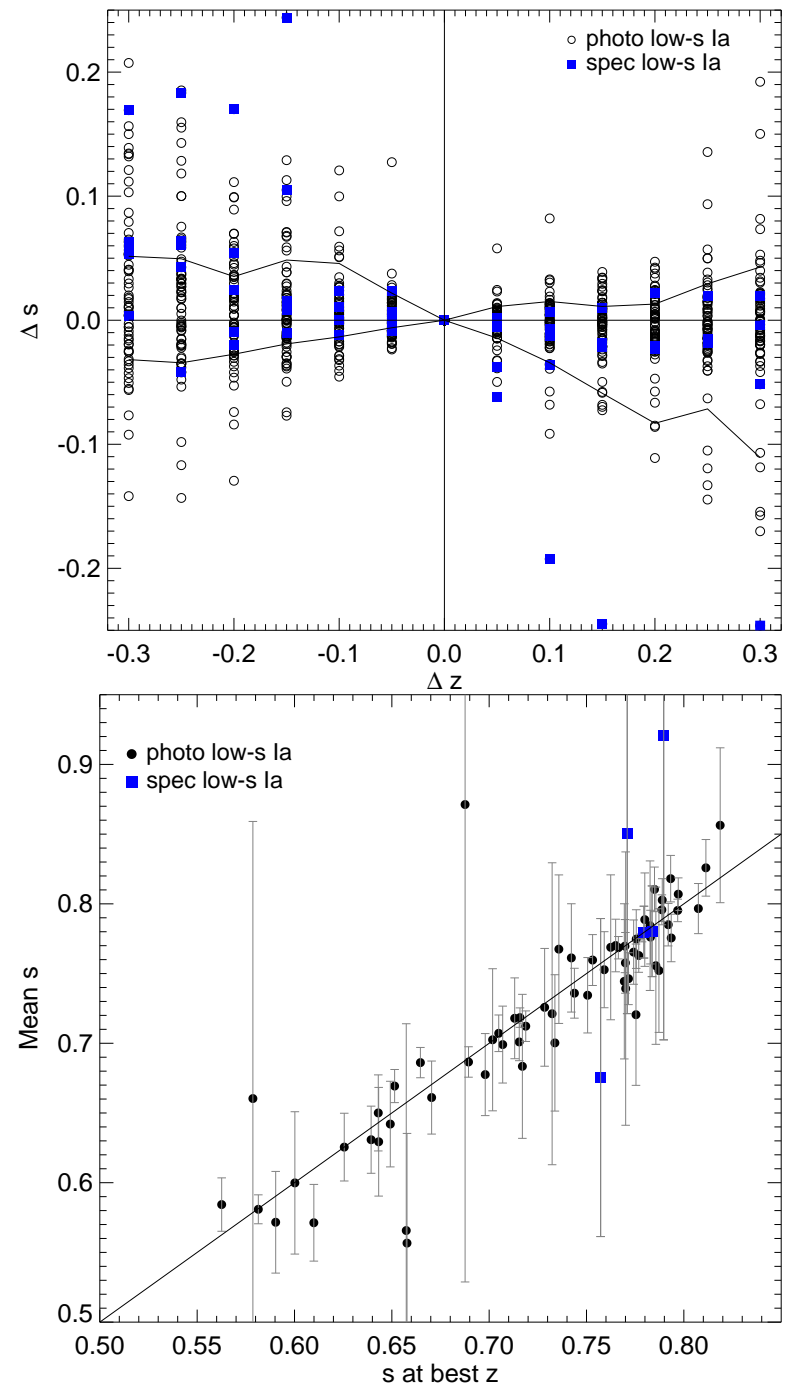

FIG. 4.- Upper: The change in fit stretch due to the deliberate variation in redshift for the low- $s$ final sample of 33.2 The blue squares represent the spectroscopically-confirmed low-s SNe Ia. The solid black lines are the standard deviation above the median $\Delta s$. Lower: The average output stretch for each SN Ia as a function of its stretch (at zero redshift offset). The error bars are the change due to the variation in redshift for each SN Ia.

Objects with $z_{\text {hostphot }}$ that have "bad fits", i.e. that do not pass all cuts of \$3.2 are not discarded, but instead the cuts are re-applied using $z_{\mathrm{SNphot}}$. If this also fails, then the object is rejected. This offers some protection against catastrophic host photo- $z$ failures. This recovers 29 confirmed SNe Ia. All known contaminants fail with both photo- $z$ values, so we are confident that this procedure still rejects non-Ias.

\subsection{Final candidates}

We perform final SiFTO (and low-s-modified estimate_sn) fits to each object to obtain the parameters of each SN and quality-of-fit values. A series of cuts are then applied to screen out objects with poorly estimated parameters or those unlikely to be SNe Ia. A summary of the applied culls and the number of objects surviving each of them can be found in Table 4 The $\chi^{2}$ culls are similar to those of P10, although we weaken the requirements slightly for $s \leq 0.8$ objects based on the study of the low- $z$ sample: 
reduced $\chi^{2}$ s of $\chi_{\text {estimate_sn }}^{2}<13$ for the overall fit, and for individual passbands $\chi_{\text {estimate_sn }}^{2}\left(r_{M}\right)<11$ and $\chi_{\text {estimate_nn }}^{2}\left(i_{M}\right)<16$, and a SiFTO cull of $\chi_{\text {SiFTO }}^{2}<11$. Additionally, we require the objects to be inside the error-snakes trained in $\$ 2$ with low- $z$ low- $s$ SNe in order to constrain their parameters (Figure 6). These cut values are derived empirically by trying to minimize the number of known contaminants that pass and minimize the number of rejected confirmed SNe Ia.

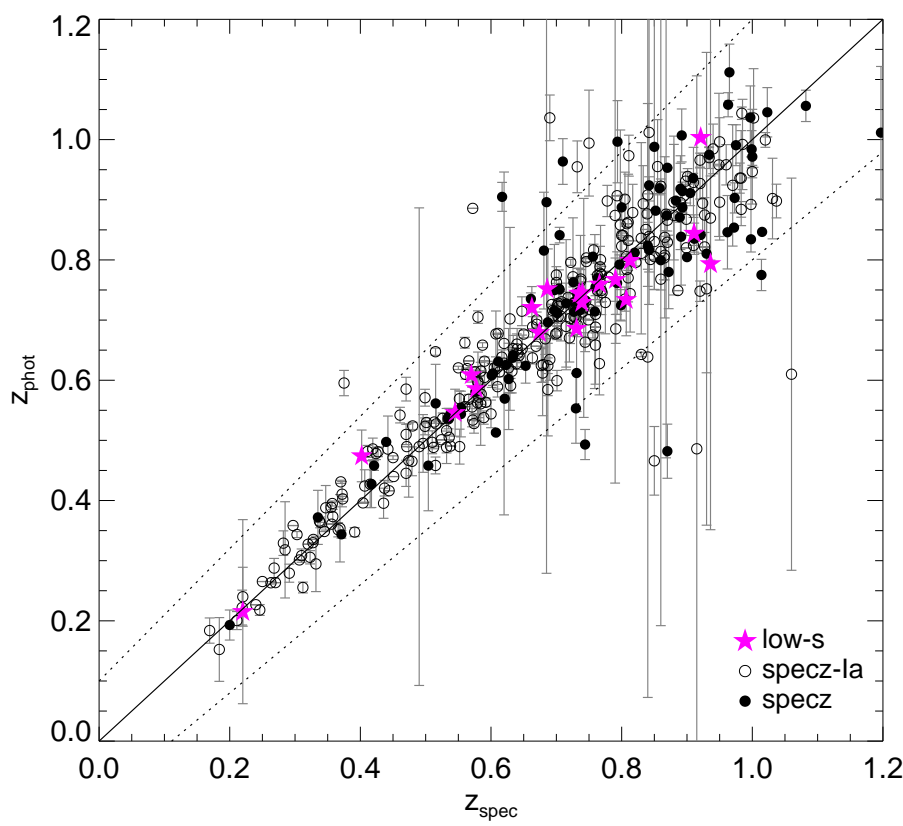

FIG. 5.- Photometric redshifts obtained from the host or from the LC fits compared to spectroscopic redshifts from the SN (open circles) or the host (filled circles). Purple stars represent $s \leq 0.8$ objects. The solid line shows 1:1 agreement and dashed lines a $10 \%$ uncertainty in $\left(1+z_{\text {spec }}\right)$. The photo- $z$ errors are either from the host or from estimate_sn.

\subsubsection{At $z \leq 0.6$}

Up to $z=0.6$, the final sample contains 161 objects, of which 126 have spectroscopic redshifts. They span the stretch range $0.6<s<1.2$, of which 18 have $s \leq 0.8$. Six of these have known spectroscopic redshifts: three are confirmed SNe Ia with $s \sim 0.8$ with no outstanding 91bg-like feature in their spectra, and the other three have spectroscopic redshifts but have too low of a contrast with the underlying host galaxy to conclusively establish the spectroscopic SN type. The final parameter distributions are shown in Figure 7 and Figure 8 . The low- $s$ photometric sample has median stretch and color of $\langle s\rangle=0.74$ and $\langle c\rangle=0.24$ (as compared to $\langle s\rangle=1.00$ and $\langle c\rangle=0.00$ for the normal-s population). The mean $\langle\Delta \mathrm{mag}\rangle=0.76 \pm 0.69$ clearly shows that our low- $s$ sample is not as extreme as the characteristic $91 \mathrm{bg}$ of $\Delta$ mag $\sim 2$ mag.

The complete final set of photo- $z$ contains $82 \%$ with host photo- $z$ and $18 \%$ with SN photo- $z$. Figure 5 shows the comparison of photometric and spectroscopic redshifts for all SN Ia candidates with spectroscopic redshift either from the SN or the host. The median precision is $\frac{|\Delta z|}{\left(1+z_{\text {spec }}\right)}=0.030$. The photo- $z$ are more accurate to $z \simeq 0.6\left(|\Delta z| /\left(1+z_{\text {spec }}\right)=0.025\right)$, where our rate study will concentrate.

The final errors in photo- $z$ are obtained from Figure 5 by taking the standard deviation around the median as a function of $\mathrm{z}$. The stretch and color errors are provided by $\mathrm{SiFTO}$, which requires a known redshift input. Stretch and color variations due to redshift uncertainties will be taken into account with a Monte Carlo simulation in $\S 4.2$.

$$
\text { 3.2.2. At } z>0.6
$$

The detection incompleteness at $0.6<z<1.0$ increases rapidly for low- $s \mathrm{SNe}$ Ia, as will be shown in next section. We still find a significant population of 44 candidates (11 with spec- $z$ from the host and 5 with low signal-to-noise spec- $z$ from the SN, 2 of which are confirmed SNe Ia of $s \sim 0.8$ and no $91 \mathrm{bg}$ features) with median stretch, $\langle s\rangle=0.76$, and color, $\langle c\rangle=-0.02$. The average color is extremely blue compared to the median color at $z<0.6$ of 0.24 confirming that we are only observing the blue tail of the low- $s$ distribution. This can be seen in Figure 9. They are not very faint objects, even for low-stretch, as shown in Figure 8. The lack of characteristic low- $s$ red objects makes it difficult to reliably estimate the low- $s$ rate at $z>0.6$ and proper corrections need to be made (see $\S 4.2$ ).

Additionally, we do find a presence of blue low- $s$ candidates at high- $z$ which are absent at lower- $z$. Such blue objects with $c<-0.05$ are neither found in the nearby nor in the $z<0.6$ SNLS samples, but represent $\sim 30 \%$ (14) of all $z>0.6$ low- $s$ objects. We do not expect all of these objects to be CC-SNe, which could potentially contaminate the low- $s$ sample with their characteristic bluer colors. As will be shown later, besides going through our SN Ia pipeline, the environment of these candidates is not characteristic of CC-SNe.

A second possibility is that this group represents another class of transients that are fast and blue. The object found by Perets et al. (2010) is faint and fastevolving but with clear different spectroscopic features and bluer colors than 91bg-like objects. It is hosted in an old stellar population as well as most of our candidates. However, our objects are not as faint (Figure 8). On the other hand, the transients found by Poznanski et al. (2010) and Kasliwal et al. (2010), suggested SN .Ia-like helium detonations of a WD, are blue and of similar brightness than ours, but evolve faster. When fit with our templates, we obtain very poor fits with $s=0.44$ and $s=0.33$, respectively, lower than our lower stretch limit for subluminous SNe Ia.

A tempting explanation is that the input photometric redshift for the LC fit is consistently wrong and affects the obtained color and stretch. This hypothesis is ruled out because 7 of these objects have spectroscopic redshift and the rest (except one) have photometric redshift from the host (with mean photo- $z$ error of $\sim 0.08$ ), which do not show any significant systematic. We have seen the small effect in the measured stretch when the redshift is varied (Figure 4). We now show the effect in the magnitude and color of photometric candidates in Figure 10. Their variation due to changes in photo- $z$ cannot account for all the bluer colors but for some of them -only if the redshifts were all consistently overestimated by +0.05 , the colors would be redder by +0.08 (and magnitudes 

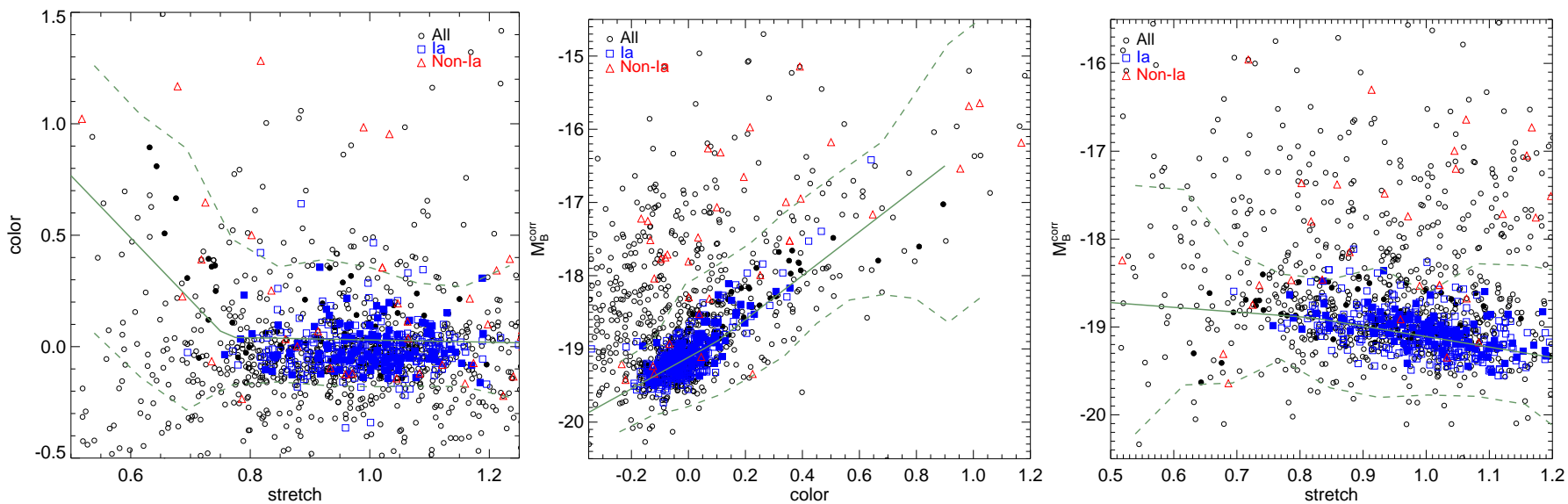

FIG. 6.- Color-stretch (left), magnitude-color (middle) and magnitude-stretch (right) for all SNLS objects passing the observation criteria (open points) and the final SN Ia photometric $z<0.6$ sample after all cuts of Table 4 (filled points). Spectroscopically-confirmed $\mathrm{SNe}$ Ia (blue squares) and non-Ia (red triangles) are shown. The lines are the best-fit relations and error-snakes found in $\$ 2$ for the local training sample, and served as additional cuts.
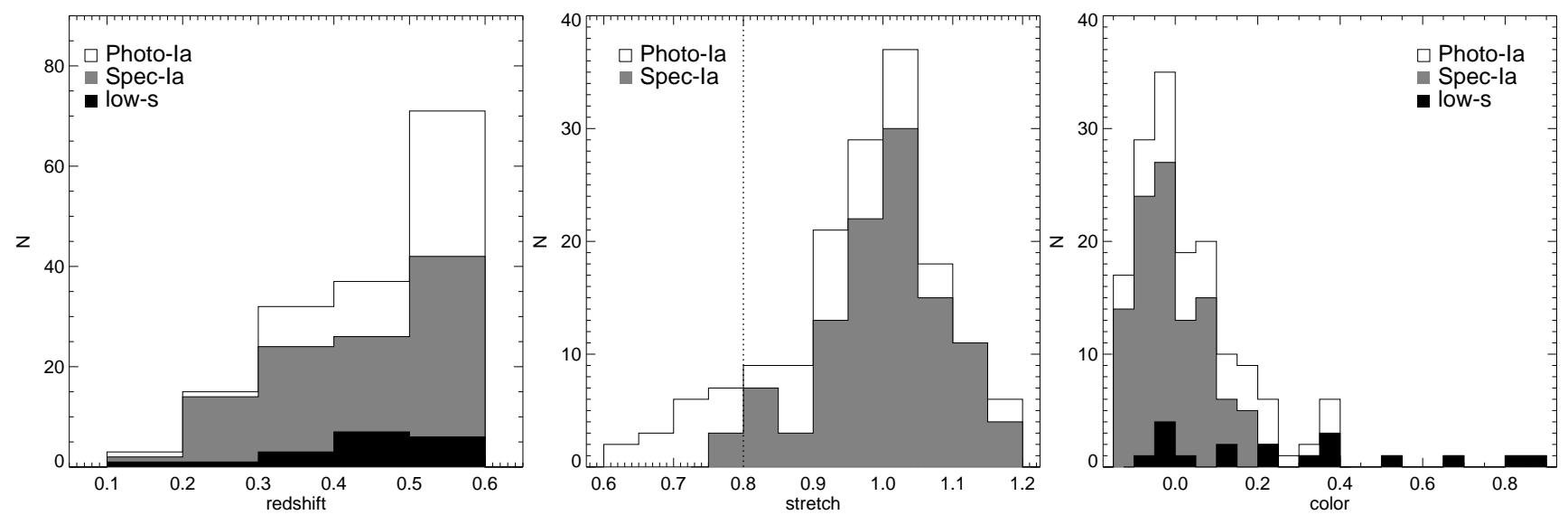

FIG. 7. - Final distributions in redshift (left), stretch (center) and color (right) of the $z<0.6$ SN Ia photometric sample including low- $s$. The white histogram is the whole population, the gray is the spectroscopically-confirmed SNe Ia and the black the low- $s$ candidates.

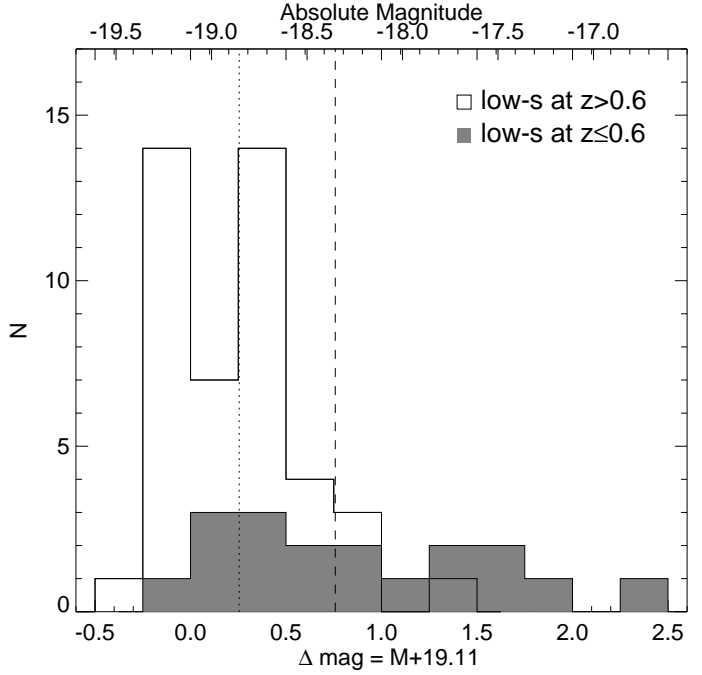

FIG. 8.- Distribution in $\Delta$ mag of the final low-s candidate sample. $\Delta$ mag $=M+19.11$ is the difference between the absolute magnitude of each SN compared to the magnitude -19.11mag of a "standard" SN Ia at $s=1$ and $c=0$ according to eq. 2 The shaded histogram shows only the low- $s$ SNe Ia at $z<0.6$.

fainter by +0.17 , whereas stretches would only change by -0.004$)$.

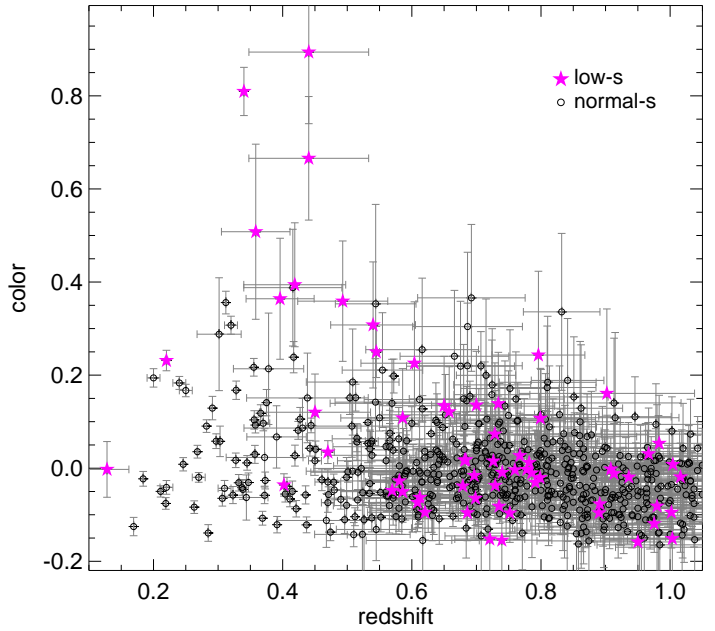

FIG. 9.- Color as a function of redshift for the final photometric SN Ia sample. Normal-s (black circles) and low-s (purple stars) are highlighted.

Photometric errors in stretch and color get larger as we probe higher redshifts. Most of the candidates found at these redshifts have stretch close to the limiting definition of $s=0.8$, so that they are consistent with being fast normal- $s$ SNe Ia. Only 3 candidates have $s+s_{\text {error }}<0.8$, 


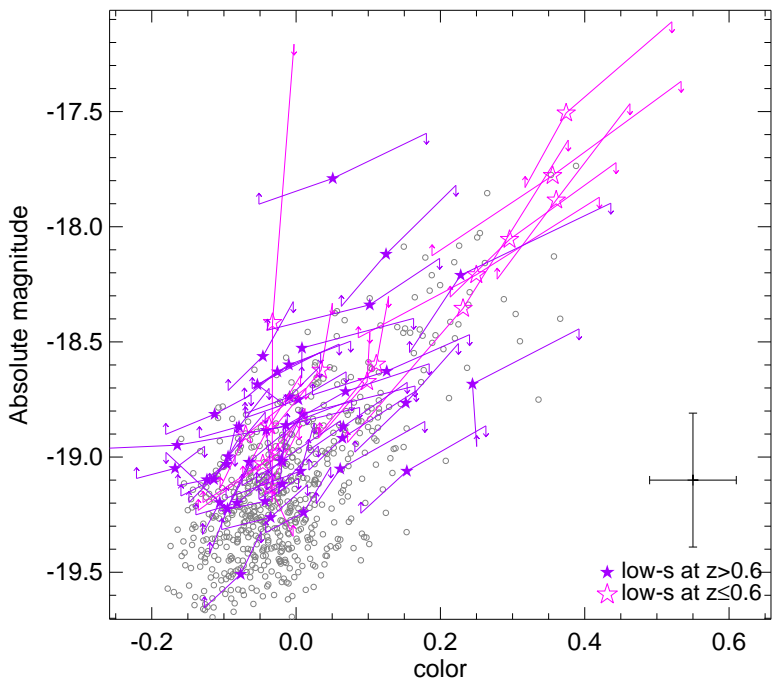

Fig. 10.- Absolute magnitude vs color for the low- $s$ SN Ia sample with photo- $z$ at $z<0.6$ (big open pink stars) and at $z>0.6$ (small filled purple stars). The dots show the normal-s sample. The upward and downward arrows show the new magnitudes and colors when varying the photo- $z$ by +0.05 and -0.05 respectively. The lines unite the same object at the three redshifts. The lower right error bars represent the typical (mean) photometric errors.

one of them with $s<0.6$. Of these,two also have $c+c_{\text {error }}<-0.05$, inconsistent with typical low- $s$ colors, and will be further discussed in 4.3. The rest can therefore be explained as the blue tail of $s \sim 0.8$ objects while the redder counterpart remains unseen due to selection effects. In the remainder of this work, we treat this group as such and correct for this selection bias when calculating the rates.

\section{LOW-STRETCH SN IA RATE EVOLUTION}

With a set of low- $s$ candidates, we proceed to calculate a volumetric rate as a function of redshift. As in $\mathrm{P} 10$, the volumetric rate, $r_{b}\left(z_{b}\right)$, for a given redshift bin, represented here by the middle of the redshift bin, $z_{b}$, is given by the sum over all $N_{b}$ candidates inside the bin:

$$
r_{b}\left(z_{b}\right)=\frac{1}{V_{b}} \sum_{i=1}^{N_{b}} \frac{\left(1+z_{i}\right)}{\epsilon_{i} \Delta T_{i}},
$$

where $\epsilon$ is the individual SN detection efficiency depending on the characteristics of each object (like redshift, stretch and color) and $\Delta T$ is the sampling time accessible to find the object, divided by $(1+z)$ to account for time dilation. $V_{b}$ is the comoving volume contained in the redshift range of the bin.

\subsection{Detection efficiencies}

The detection efficiencies $\epsilon_{i}$ account for observing biases measuring the rate. They depend on numerous factors and are specified as a function of the SN characteristics such as redshift, stretch and color (along with field and year of observation). The efficiencies used here are described in P10 and Perrett et al. (2010b), and are based on a Monte-Carlo study of 2.4 million artificial SNe Ia injected into the actual SNLS images. This approach avoids the need to make assumptions about temporal and spatial data coverage.

The artifical SNe are generated using uniform distributions of redshift and stretch (down to $s=0.5$ ), and the color is determined using a relation like Eq. 1, including an intrinsic scatter around the stretch-color relation (a Gaussian noise with dispersion $\sigma=0.04$ added independently to $U$ and $V$ ). The peak magnitudes are generated from the luminosity-stretch-color relation, as in Eq. 2 . and the redshift and assumed cosmology, plus an additional unmodeled magnitude dispersion from a Gaussian distribution with an intrinsic scatter of $\sigma_{i n t}=0.15$. The $\mathrm{LC}$ is generated from integrating, at a given epoch and through the filter functions, the SN Ia spectral templates scaled for the $B$-magnitude and corrected for $U-B$ and $B-V$ colors, Milky Way and host extinction. The artificial SN is then inserted into each $i_{M}$ image obtained by the SNLS. The images are then processed through the same pipeline used in the real-time SN discovery, and the recovered fraction is used to derive the efficiency as a function of various parameters, including redshift, stretch, color, observing season and field (see a more detailed description in Perrett et al. 2010b). The low$s$ candidates, which are faint, red and have a rapid rise and fall, are more difficult to detect and therefore have a lower efficiency, so are accordingly given a higher weight when calculating the rates.

The efficiencies are calculated in bins of width $\Delta z=$ $\Delta s=\Delta c=0.1$. The measured efficiencies, linearly interpolated according to each SN, are shown in Figure 11 It can be seen that the mean efficiencies are lower for the low- $s$ sample up to $z \sim 0.6$. Above $z=0.6$, the mean efficiencies seem to approach the normal- $s$ sample. At these redshifts they become low for all but the bluest low- $s$ objects. This leads to a higher fraction of low- $s$ blue objects with very few red ones recovered, as found in $\S 3.2$. We show the expected efficiency distribution for the missing red, low- $s$ SNe Ia of an unevolving color distribution as green squares in Figure 11 based on the observed low- $s$ color distribution at low redshift from 87 . The rates at $z>0.6$ will need to be corrected for these missing objects (see next section). We note that the efficiencies shown in Figure 11 are the raw efficiencies -the recovery fraction from the simulated $\mathrm{SNe}_{-}$- without the observational constraints of section $\S 3$ nor the sampling time correction that depends on each field and season. These corrections can make the actual efficiencies much lower (see P10). For instance, one of our low- $s$ candidates at high- $z$ with $z=0.80, s=0.63$ and $c=0.24$ has a raw efficiency of 0.54 but with observational constraints and sampling time this efficiency is only 0.18 , which explains why these objects are hard to observe.

\subsection{SN Ia Rates and Monte-Carlo simulations}

We calculate a rate evolution, $r_{\text {meas }}$, shown in Column 5 of Table 5 solely based on Eq. 3 and with the efficiencies from $\delta 4.1$. We use bins of $\Delta z=0.2$ due to the low sample statistics (the last $z$-bin is only 0.1 wide as no low- $s$ candidate exists beyond $z=1$ ). The measured errors in stretch and color from the LC fit, and especially the errors in the photometric redshift of each candidate, can lead to uncertainties in the rate measurement. We account for these via a Monte-Carlo (MC) simulation that randomly shifts the photometric redshift according to its error (for those SNe Ia with photo- $z$ ). For each redshift, a corresponding LC fit provides new parameters and its covariance matrix. This latter is used to generate a new random set of stretch and color using standard tech- 


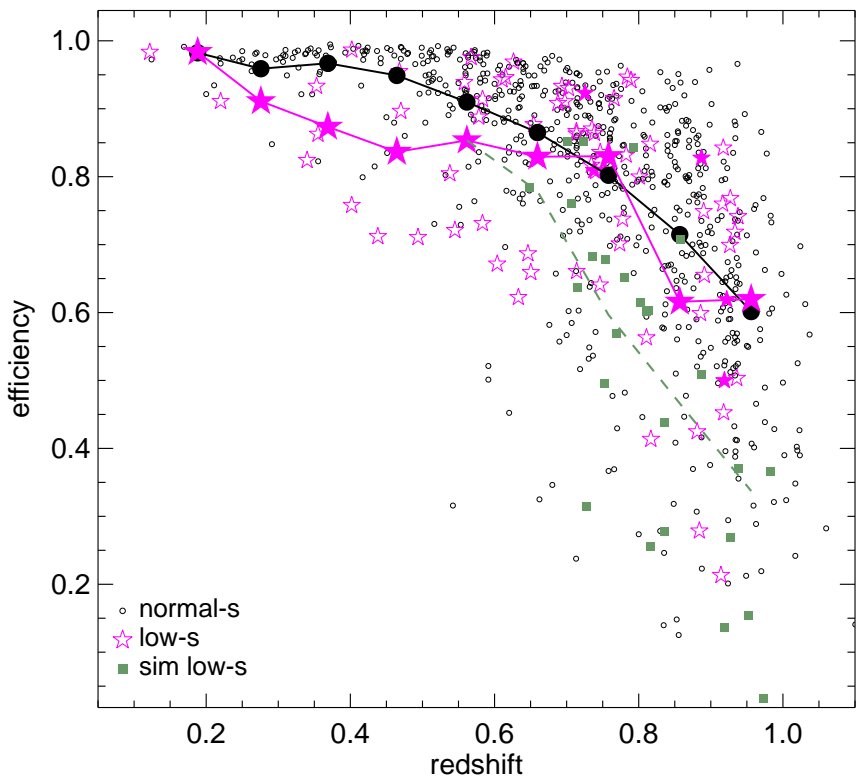

FIG. 11.- Efficiencies calculated by P10 for the SN Ia photometric sample for normal-s (black circles) and low- $s$ (purple stars) populations. For objects where no direct efficiency was found (small filled stars), the closest match in color was used. Mean values in redshift bins for normal-s and low- $s$ populations (big filled circles and stars) are shown. Additionally, simulated low- $s$ objects for $z>0.6$ bins (green squares) based on the $z<0.6$ color distribution and respective mean (green dashed line) are shown for comparison. Note that these efficiencies do not include the observation criteria of section $\S 3$ and sampling time correction of the different fields observed.

niques (James \& Roos 1975). We recalculate the rates from Eq. 3 for each iteration based on the randomized values, and use the median value over the 1000 realizations to determine the final rate, $r_{\mathrm{MC}}$. We estimate the error from the variance of the rates in each bin (and call them "MC" errors shown in Column 6 of Table 5 and Figure 12) and add it in quadrature to the weighted $1 \sigma$ Poisson statistical errors in Figure 12 (confidence levels calculated using the Clopper-Pearson method used for example in Gehrels 1986). The error simulation differs from P10, as the SNLS does not possess a known spectroscopic sample of low- $s$ SNe Ia from which we could obtain parameter distributions. Instead, we treat each SN individually by randomly drawing parameters according to its errors and covariances. Although the precise details of our simulation differ from P10, a SN Ia rate evolution within the errors is obtained.

Additionally, we have seen in the previous section that we do not find red objects in the higher- $z$ bins. This selection bias results in underestimated rate values at high- $z$, which are not corrected by the efficiencies of the bluer objects found. There is a lack of objects in certain red color bins, so that no efficiency can account for them. To measure this effect, we take the low- $s$ distribution of color in an underestimated high- $z$ bin (for each MC iteration), we normalize this distribution to the known color distribution at $z<0.6$ (right Figure 77), which has already been corrected for the efficiencies of each $\mathrm{SN}$, and then generate fake $\mathrm{SNe}$ in the missing color bins. The normalization is done with the two bluest matching bins. Figure 13 shows an example for $0.7<z<0.9$. The green open histogram is the final corrected histogram from the initial observed diagonal-shaded distribution. The cor- recting factor for this case is $f_{b, \text { corr }}=1+\frac{N_{b} \text { (fake) }}{N_{b}}=1.9$, where $N_{b}$ (fake) is the number of generated $\mathrm{SNe}$ in the bin and $N_{b}$ is the observed number, so that the corrected rate is $r_{b, \text { corr }}=r_{b} \times f_{b \text {,corr }}$. The final correcting factors from the median of all MC iterationns, summed Poisson (from the number of objects used in the bins used to normalize both distributions) and MC errors, as well as the resulting corrected rates are shown in the last two columns of Table 5. In Figure 12, the corrected rates are shown as stars.

Finally, errors in the efficiencies can affect the rate values. As explained in P10, those come mainly from systematic errors in the underlying assumed $\Delta$ mag distribution of SNe Ia, the distribution of residuals between the absolute magnitude of a fiducial $s=1 \mathrm{SN}$ Ia and the magnitudes of observed SNe Ia after correction for stretch and color. To check for this systematic error, we allow for random efficiencies in the MC simulation calculated from a range of $\sigma_{i n t}=0.12-0.15$ in the $\Delta \mathrm{mag}$ distribution, obtaining results that agree very well with the rates of Figure 12

\subsection{Contamination uncertainties}

An important systematic error comes from corecollapse SNe mimicking the LC of low- $s$ SNe Ia. In P10, a missclassification fraction of less than $\leq 3 \%$ was found for the normal-s population. We expect this fraction to be higher for low- $s$ SNe Ia due to two effects. First, they are dimmer, and more easily confused with the intrinsically fainter CC-SNe at a given redshift. Second, as we probe higher redshift ranges, CC-SNe at low- $z$ might masquerade as low- $s$ SNe Ia at higher- $z$ since we do not have spectroscopic redshifts for all candidates. SNe II have characteristic LCs that are very different from SNe Ia and present less danger of contamination. $\mathrm{SNe} \mathrm{Ib} / \mathrm{c}$, on the other hand, represent a smaller fraction of CC-SNe, $30 \%$ according to Cappellaro et al. (1999); Smartt et al. (2009) and $25 \%$ according to Li et al. (2010a), but are more similar to SNe Ia and will be the most probable contaminants.

The first error -where the photo- $z$ is correct, yet the fit of a SN Ia template manages to pass the qualityof-fit cuts- can be examined using the training sample (as in 22). We found a 6\% CC-SN missclassification rate, all $\mathrm{SNe} \mathrm{Ib} / \mathrm{c}$. This fraction can be propagated to higher redshift using the SN Ia rate evolution of P10, $R_{I a}(z) \propto(1+z)^{\alpha_{I a}}$ with $\alpha_{I a} \simeq 1.91$, and a CC-SN rate evolution reflecting the star formation rate from Hopkins \& Beacom (2006),$R_{C C} \propto(1+z)^{\alpha_{C C}}$ with $\alpha_{C C} \simeq 3.6$. We assume a ratio of SNe Ia to CC-SNe of $\sim 0.22$ at $z=0.3$ from Bazin et al. (2009), which translates to a local ratio of $\sim 0.39-$ as compared to $\sim 0.48$ found by Cappellaro et al. (1999) and 0.24 from Li et al. (2010a) - . The percentage fraction of contamination as a function of $z, f_{\text {cont }}(z)$, can then be described as:

$$
f_{\text {cont }}(z)=\frac{N_{\text {passcont }}(z)}{N_{\text {pass }}(z)}=\frac{1}{1+0.39 r(1+z)^{\alpha_{\mathrm{Ia}}-\alpha_{\mathrm{CC}}}},
$$

where $r=0.88 / 0.06$ is the cull passing efficiency (the probability of a SN passing all selection criteria) ratio of Ia with respect to CC, assuming it does not evolve with $z$. This equation allows us to get a crude estimate 


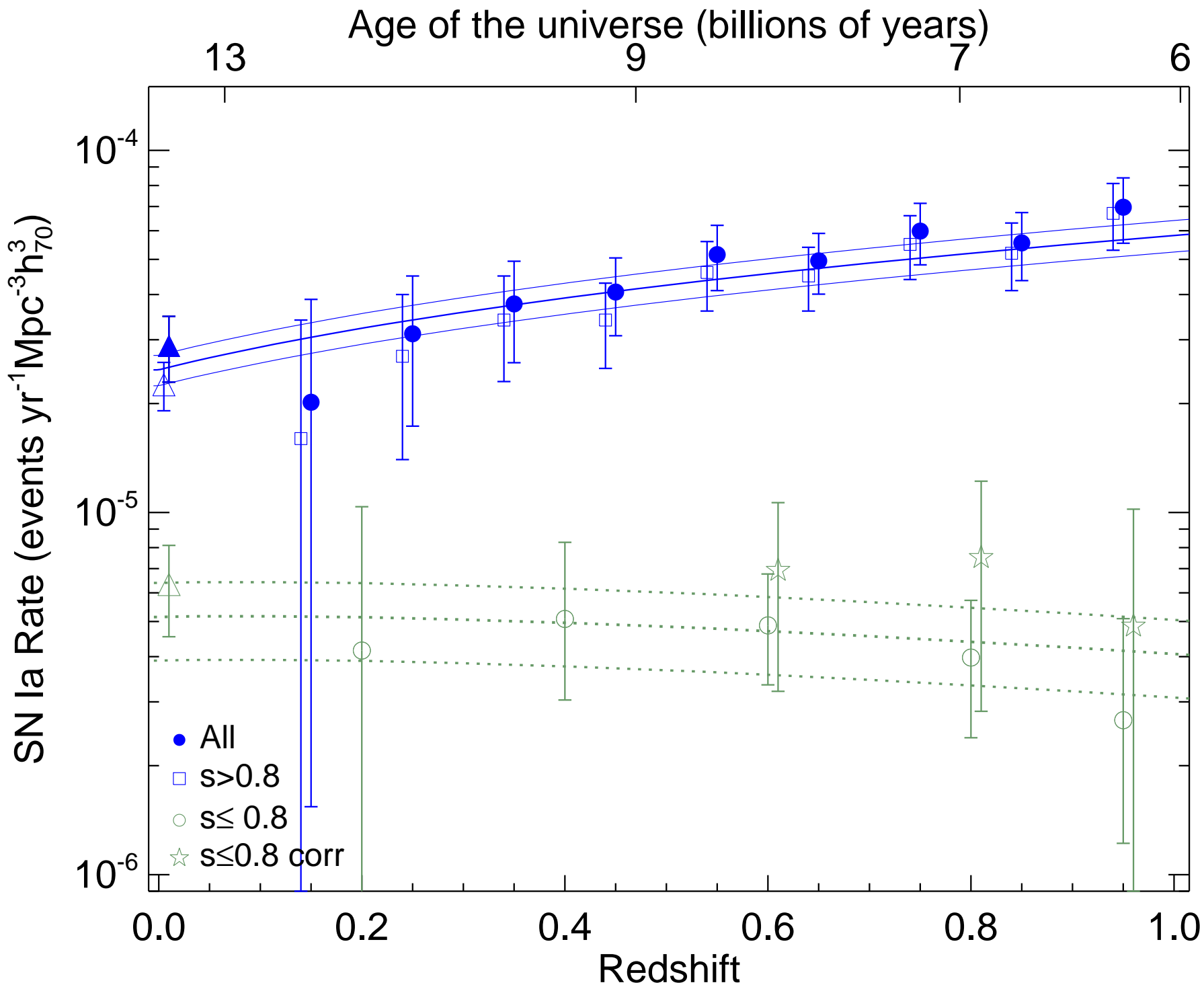

FIG. 12.- Volumetric rate of low- $s$ SNe Ia as a function of redshift (green open points) compared to the normal- $s$ rate (blue squared points) of P10. The total summed rate (blue filled points) is shown with a little $z$-offset for clarity. The stars represent the corrected high- $z$ low- $s$ rate calculated from real and simulated objects drawn from a distribution scaled to the $z<0.6$ color distribution. The filled triangle is the total nearby SN Ia rate from $\mathrm{Li}$ et al. $(2010 \mathrm{~b})$ and the open triangle is the low- $s$ rate estimated from a $s<0.8$ fraction of it. The solid lines are the best $A+B$ model and uncertainties found in P10. The dashed lines are the best A-model and uncertainties found in this study through a fit of only SNLS $z<0.6$ bins: $A=(1.05 \pm 0.28) \times 10^{-14} \mathrm{SNe} \mathrm{yr}^{-1} M_{\odot}^{-1}$.

of the number of contaminants that would pass all cuts, $N_{\text {passcont }}(z)$. By dividing the expected fraction of contaminants per $z$-bin by the mean weight of low- $s$ SNe found therein (per MC simulation), we translate this into a rate error lower than $20 \%$ for $z<0.6$ up to as high as $30 \%$ at $z \simeq 0.9$. The estimates are sensitive to the power law of the rate evolution, the initial fraction of SNe Ia to CC-SNe and the local missclassification fraction of $6 \%$. Increasing this latter fraction to $10 \%$ or using SN Ia to CC-SN local ratio of $0.241_{-3.8}^{+3.7}$ by Li et al. (2010a) instead, we obtain contamination percentages up to $30 \%$ for $z<0.6$ and $40 \%$ at $z \simeq 0.9$. This latter rate error from contamination is added in Table 5] and we believe it to be an upper limit. The contamination error is smaller than the combined statistical and MC errors at $z<0.6$ but becomes comparable at higher- $z$. One should note that the missclassification error increases with redshift and would only make the rate higher than the actual one.

The second source of contamination comes from uncertainties in the photo- $z$. Incorrect redshift estimates can cause low- $z$ CC-SNe to simulate low- $s$ SNe Ia at high$z$. This effect can be tested by running all spectroscopic objects through our selection criteria with their calculated photo- $z$ instead of the spec-z. By doing this, we retain most of our candidates with a similar fit stretch and, most importantly, we still reject all the CC-SNe as contaminants (even though their photo- $z$ was calculated with SN Ia templates and can occasionally be very different from the spec-z).

Perhaps the most concerning signature of contamination is the low- $s$ SN Ia sample at $z>0.6$. These have rather blue colors and could be CC-SNe instead. As an additional test, we analyzed the host galaxy properties (Figure 14). In general these objects are found in massive and passive galaxies, not characteristic of CC-SNe. 


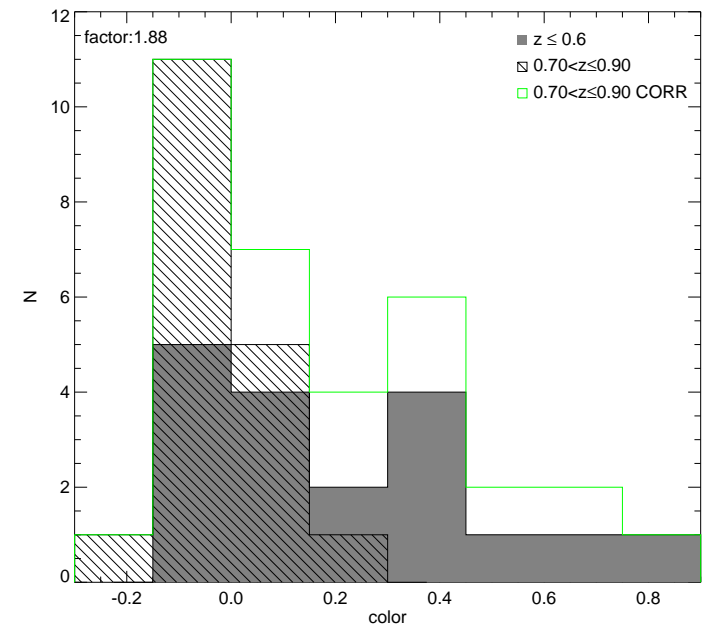

FIG. 13. - Example of how the observed color distribution for low- $s$ at $0.7<z<0.9$ (diagonal-shaded) is corrected to match the efficiency-corrected low- $s$ color distribution at $z<0.6$ (shaded). The final corrected color distribution for that $z$-bin (open histogram) has $f_{b}=1.88$ more $\mathrm{SNe}$ than the original observed one.

This provides evidence that most of these candidates, including the $z>0.6$ population, are likely the actual blue tail of the low- $s$ SN Ia distribution, as mentioned earlier.

Nevertheless, two of these objects, 05D3hp (with $s=$ $0.59 \pm 0.04$ and $c=-0.16 \pm 0.09$ ) and $07 \mathrm{D} 2 \mathrm{bc}$ (with $s=0.72 \pm 0.04$ and $c=-0.13 \pm 0.03$ ), have unusually blue colors at very low-stretch. The host of 05D3hp is small $\left(M=10^{7.9} M_{\odot}\right)$ and star-forming (SFR/mass = $10^{-9.8} M_{\odot \mathrm{yr}^{-1}}$ ) and points rather towards a $\mathrm{CC}$ event imitating a low- $s$ SN Ia. 07D2bc happened also in a star-forming $\left(\mathrm{SFR} /\right.$ mass $\left.=10^{-10.0} M_{\odot} \mathrm{yr}^{-1}\right)$ but rather massive galaxy $\left(M=10^{11.0} M_{\odot}\right)$. If these two objects are CC-events, they would constitute a $\sim 5 \%$ contamination fraction. Although it is dangerous to assume the local star formation from general galaxy properties, if we were to assume that all events in "burst" hosts with SFR/mass $<-9.5 M_{\odot} \mathrm{yr}^{-1}$ are contaminants, then $\sim 30 \%$ of the $z>0.6$ objects are missclassified $(\sim 25 \%$ at $z \leq 0.6$ ), in agreement with our generous estimates.

\section{DISCUSSION}

We now turn to the interpretation of the low- $s$ SN Ia rate in the light of suggested rate models, and compare it to different stretch populations. We investigate the empirical two-component rate evolution model of Mannucci et al. (2005) and Scannapieco \& Bildsten (2005) but also study simple delay-time distributions (Dahlen \& Fransson 1999; Strolger et al. 2004) for SN Ia progenitors. A local low- $s$ rate estimate based on nearby studies helps constrain the models.

\subsection{Low-z subluminous rate}

Recently, Li et al. (2010b) have calculated a lowredshift SN Ia rate and a corresponding $17.9_{-6.2}^{+7.2 \%}$ fraction of 91bg-like objects (Li et al. 2010a). To obtain a rough local rate estimate of low- $s$ SNe Ia defined according to our study, i.e. $s \leq 0.8$, we use their luminosityfunction sample (Table 3 of Li et al. 2010a) and directly fit objects for which we have photometric data (48 of 74) with SiFTO. For the rest we fit instead their closest $R$-band LC template match (column 11). Using the completeness-corrected numbers (column 9), we obtain 16.4 objects with $s \leq 0.8$, or a $21.9_{-5.4}^{+6.9} \%$ fraction. This fraction is complete within a volume of $80 \mathrm{Mpc}$ and translates to a low- $s$ rate of $(6.3 \pm 1.8) \times 10^{-6} \mathrm{yr}^{-1} \mathrm{Mpc}^{-3} h_{70}^{3}$ based on the local rate of Li et al. (2010b) (adjusted for $h_{70}=1$ ) and including the uncertainty from the Poisson error. We note that by using the whole low- $z$ training sample of 42 the 41 low- $s$ objects correspond to a $22 \%$ fraction of the total, in good agreement.

This rate estimate is based on small sample statistics and suffers from the biases that arise from a distancelimited host-targeted survey, where the rate of $\mathrm{SNe}$ is converted to a volumetric rate via the galaxy luminosity function. This can lead to systematic errors, as brighter galaxies may contain more $\mathrm{SNe}$ Ia of a certain type (as is expected for low- $s$ ). However, a local low- $s$ rate estimate is a valuable tool for comparison to the high- $z$ rates.

\subsection{Power-law evolution}

The low- $s$ SN Ia rate, as opposed to the normal- $s$ population, does not seem to increase with redshift and follow the star formation history (SFH) trend found in P10 (Figure 12). If we fit a simple power-law, $r \propto(1+z)^{\alpha}$, to the SNLS rate to $z=0.6$ and the low- $z$ estimate, we obtain $\alpha=-0.90 \pm 1.38$, smaller than the value of the normal-s population found by P10 $\left(\alpha_{\text {normal }}=\right.$ $1.91 \pm 0.24$ ). This means that an evolution similar to the normal-s population is rejected with $\sim 94 \%$ probability. If we include the corrected $z>0.6$ SNLS points, we find a higher slope more consistent with zero: $\alpha=-0.34 \pm 1.41$.

\subsection{Delayed component model}

It is known that $\mathrm{SNe}$ Ia occur in both star forming and non-star forming regions, whereas low- $s$ objects are usually found in passive ones. In the model of Mannucci et al. (2005); Scannapieco \& Bildsten (2005), two populations are considered: a "prompt" component for SNe exploding in $0.1 \mathrm{Gyr}$ or less that is proportional to the star formation history, $\dot{M}(z)$ and a "delayed" component for events with higher delay times that is proportional to the host mass, $M(z)$. It is described as:

$$
S N R_{\mathrm{Ia}}(z)=A M(z)+B \dot{M}(z),
$$

where A and B are scale factors for both components. The delayed model for low- $s$ SNe Ia is supported when one looks at the host properties of the low-s sample. CFHT Legacy Survey host galaxy broadband photometry can be fitted to several template galaxy SEDs using PEGASE/ZPEG (Le Borgne \& Rocca-Volmerange 2002) to determine average galaxy star formation rates (SFRs), stellar masses and photometric redshifts (Sullivan et al. 2006b). The properties of SNe Ia are compared to their host type (see Figure 14) to investigate the behavior of low- $s$ objects in contrast to the normal- $s$ population.

We use the specific star formation rate (sSFR), the star formation per unit stellar mass, as the indicator of star formation activity, and separate the host galaxy sample in passive galaxies, $\log \left(\operatorname{sSFR}\left(\mathrm{yr}^{-1}\right)\right)<-11.5$, weak star formers, $-11.5 \leq \log \left(\operatorname{sSFR}\left(\mathrm{yr}^{-1}\right)\right) \leq-9.5$, and strong star formers $\log \left(\operatorname{sSFR}\left(\mathrm{yr}^{-1}\right)\right)>-9.5$, where SFR refers to the average star formation over the last 

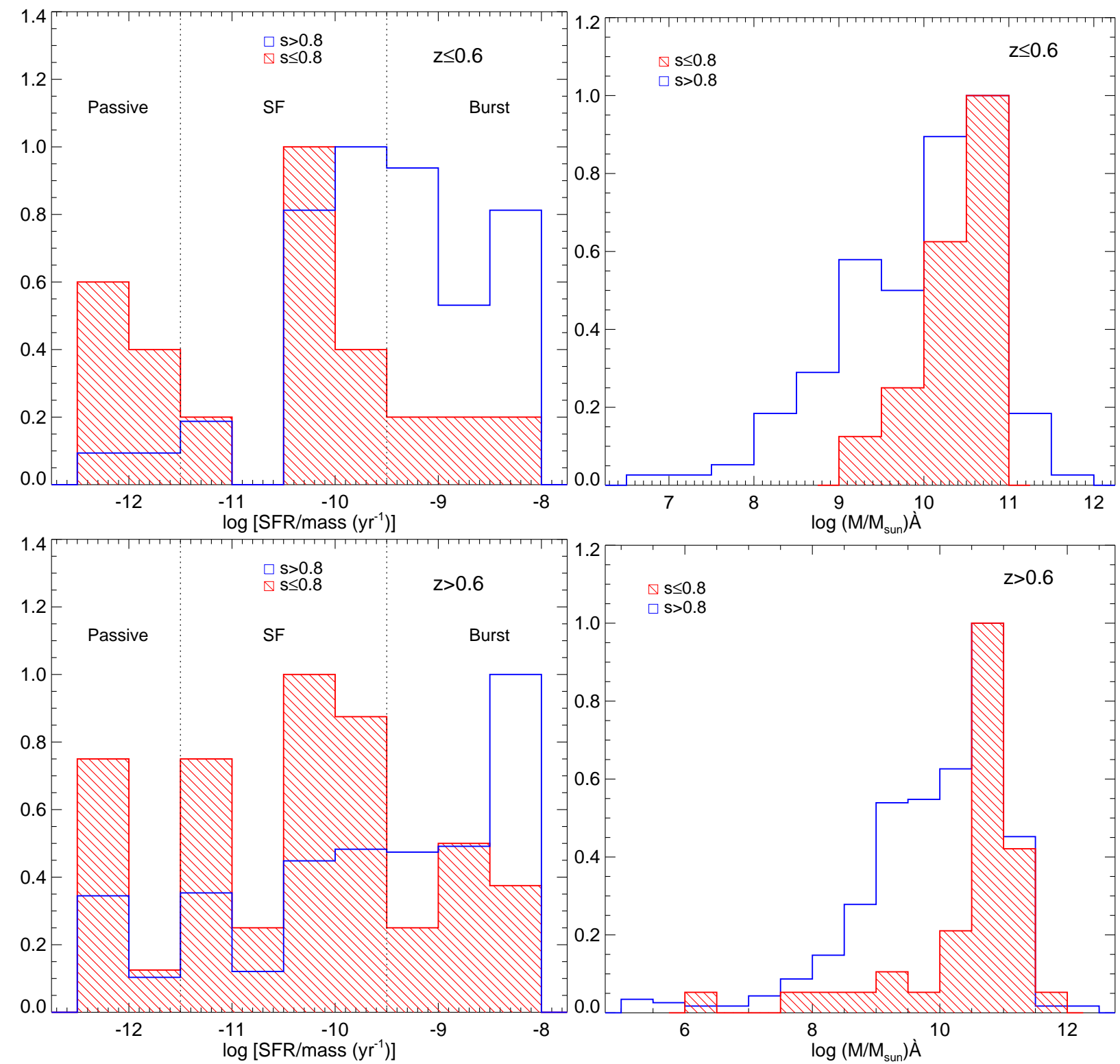

FIG. 14.- Host properties for $z<0.6$ (upper) and $z>0.6$ (lower) SN Ia candidates. Left: Normalized specific star formation (star formation per unit mass) distribution divided in normal-s (blue) and low-s (red) populations. The probability that the two populations are drawn from the same distribution (KS-statistic) is less than $\sim 0.5 \%$ for both redshift ranges. Right: Normalized host stellar mass distribution per stretch range, normal-s (blue), and low-s (red). The KS-statistic is below $\sim 7 \%$ for both redshift ranges.

0.5 Gyr. This approach ignores important internal variations of SF in the galaxy, as shown in Raskin et al. $(2009 \mathrm{~b})$, and provides only a general estimate of the SF. The sSFR distribution for two different stretch ranges (left Figure 14), normal-s and low- $s$, provides clear evidence that the low- $s$ population is less dependent on star formation. Neill et al. (2009) find that no local low$s$ SN Ia is found in a host with $\log \left(\operatorname{sSFR}\left(\mathrm{yr}^{-1}\right)\right)>-9.5$. Here, we corroborate their results for high- $z$ objects finding that only $3(17 \%)$ at $z<0.6$, and 11 (25\%) at higher redshift, have higher mass-weighted star formation rates.

Moreover, the host mass distribution for these stretch ranges (Figure 14 right) shows clearly different distributions, in which low- $s$ SNe Ia occur almost solely in massive hosts. We extend the results of Neill et al. (2009) to the distant universe: low- $s$ SNe Ia are found mostly in hosts with masses above $\sim 10^{9.5} M_{\odot}$. For all cases, the distribution of host-galaxy mass for the two populations, normal- $s$ and low- $s$, have a low two-sided KolmogorovSmirnov (KS) statistic, indicating a small probability that they are randomly drawn from the same distribution.

The above relations of SN Ia properties with host characteristics demonstrate that previous findings extend to the extreme of the SN Ia population at $z>0.1$, and at the same time are a confirmation that the observed photometric low-s sample obtained is real and not strongly contaminated from core-collapse events that are only found in late-type galaxies.

They also provide additional support to model the low- $s$ SN Ia rate as just an $A$-component. Fitting the latter to the local and SNLS and rates up to $z=0.6$ provides $A=(1.05 \pm 0.28) \times 10^{-14} \mathrm{SNe} \mathrm{yr}^{-1} M_{\odot}^{-1}$ and is shown in Figure 12. If we fit both components, we 
obtain $A=(1.29 \pm 0.66) \times 10^{-14} \mathrm{SNe} \mathrm{yr}^{-1} M_{\odot}^{-1}$ and $B=(-0.27 \pm 0.64) \times 10^{-4} \mathrm{SNe} \mathrm{yr}^{-1}\left(M_{\odot \mathrm{yr}^{-1}}\right)^{-1}$. When we include the corrected SNLS $z>0.6$ points, we obtain $A=(1.14 \pm 0.61) \times 10^{-14} \mathrm{SNe} \mathrm{yr}^{-1} M_{\odot}^{-1}$ and $B=$ $(0.03 \pm 0.62) \times 10^{-4} \mathrm{SNe} \mathrm{yr}^{-1}\left(M_{\odot} \mathrm{yr}^{-1}\right)^{-1}$. In the latter two cases, $B$ is close to zero $(B<0$ is an unphysical result) suggesting that the mass component dominates over the component proportional to the star formation history.

Comparing our delayed component (for $z<0.6$ ) with the normal SN Ia population, the ratio of this value to the one in $\mathrm{P} 10$ is $\frac{A_{\mathrm{low}-s}}{A_{\mathrm{P} 10}} \simeq 30 \%$, which is particularly important at low- $z$, where the delayed component becomes dominant. This ratio sets a constraint on the fraction of low- $s$ progenitors.

\subsection{Delay-time distributions}

Ideally, the rate should be able to help constrain the SN Ia delay-time distribution as follows:

$$
\operatorname{SNR}_{\mathrm{Ia}}(t)=\nu \int_{t_{F}}^{t} \operatorname{SFR}\left(t^{\prime}\right) \operatorname{DTD}\left(t-t^{\prime}\right) d t^{\prime},
$$

where $t^{\prime}$ is the age of the universe, $t_{F}$ the time at which the first stars formed (effectively zero for the SNLS), $\operatorname{DTD}\left(t_{d}\right)$ is the delay-time $\left(t_{d}\right)$ distribution and $\nu$ is the number of SNe Ia that explode per unit stellar mass formed. This number is related to the progenitor mass range and IMF, $\Psi(M)$, as well as the efficiency $\eta$ or fraction of white dwarfs that explode as SNe Ia, and the range of $\mathrm{WD}$ progenitor masses, $M_{\mathrm{WD}}^{\text {low }}$ and $M_{\mathrm{WD}}^{\mathrm{up}}$ :

$$
\nu=\eta \frac{\int_{M_{\mathrm{WD}}^{\text {low }}}^{M_{\mathrm{WD}}^{\mathrm{up}}} \Psi(M) d M}{\int_{0.1 M_{\odot}}^{125 M_{\odot}} M \Psi(M) d M}
$$

Eq. 6] can set constraints on the progenitors through the delay-time distribution if the SFH is known. We assume here the parameterization of Hopkins \& Beacom (2006) for a SalA IMF $(\mathrm{h}=0.7)$ :

$$
\mathrm{SFH} \propto \frac{(0.017+0.13 z) h}{1+(z / 3.3)^{5.3}} .
$$

A simple parametrization power-law DTD $\propto t^{n}$, has been used by several authors to model the $\mathrm{SN}$ Ia rate. $n \simeq-1$ is obtained in the $\mathrm{SN}$ Ia rate studies made by Totani et al. (2008); Maoz et al. (2010a,b); Maoz \& Badenes (2010) and is consistent with the DD scenario. Pritchet et al. (2008) obtain a different powerlaw of $n \simeq-0.5$ investigating the $\mathrm{SN}$ Ia rate as a function of sSFR in the SNLS. It is interesting to see how the sub-sample of low- $s$ behaves, in particular as the inferred DTDs tend to have a low tail at higher delay-times (after a sharp initial peak) where the subluminous SNe Ia are expected to lie.

By fitting this model to the local and SNLS data up to $z=0.6$, we obtain $n=-0.93 \pm 2.13$ reflecting the large uncertainties in the rates. With the corrected $z>$ 0.6 rates, the power-law becomes $n=-2.67 \pm 6.98$ (see Figure 15). This is accomplished by increasing the other free parameter, the scale $\nu$, by an order of magnitude such that many more SNe Ia happen at early times, in order to compensate for the steady rates at higher- $z$.
We also use the simple empirical models employed in Strolger et al. (2004): an exponential function motivated by the double-degenerate scenario (Tutukov \& Yungelson 1994) and a Gaussian function motivated by a DTD with a specific delay-time (Dahlen \& Fransson 1999). The Gaussian distribution can be a proper approach for sub-groups of SNe Ia, such as the low- $s$ one, but not for the entire population, which has been shown to be either bimodal or slowly declining in time. We use the formalism of the unimodal skewnormal DTD of eq.6 of Strolger et al. (2010) as a generalization of these models encompassing a wide variety of shapes and distributions.

The model of Strolger et al. (2010) has three independent variables, We obtain for the local and $z<0.6$ rates: $\xi=6.4 \pm 3.8, \omega=1.6 \pm 5.2$ and $\alpha=-81.2 \pm 26400$, which translate to a Gaussian behaviour of characteristic delaytime $\tau=(5.11 \pm 5.65)$ Gyr and width $\sigma=0.94 \pm 3.16$. This fit is shown in Figure 15. It indicates that low$s$ SNe Ia come from progenitors 3-10 Gyr old following a single gaussian-shaped distribution. Such delay-times correspond to masses of $1.5-2.5 M_{\odot}$ using $t \sim m^{-2.5}$. This sustains the argument that low- $s$ SNe Ia come exclusively from a delayed channel of SNe Ia. Such estimates are higher than the lower delay-time limit obtained by Schawinski (2009) based on individual SN Ia studies in early-type galaxies with optical and UV-data. On the other hand, they agree with Brandt et al. (2010) who find that SDSS SNe Ia with $s<0.92$ have delay-times of $\gtrsim 2.4 \mathrm{Gyr}$. Fitting all points, corrected SNLS and low- $z$, we obtain $\tau=(5.88 \pm 4.84)$ Gyr and $\sigma=0.15 \pm 2.41$ $(\xi=5.8 \pm 3.6, \omega=0.2 \pm 4.0$ and $\alpha=0.9 \pm 17300)$ instead. This approximates to a much narrower Gaussian spanning 5.3-6.3 Gyr $\left(1.95-2.10 M_{\odot}\right)$, time at which all low- $s$ SNe Ia are created. This explains the sharp drop in the modeled rate at $z \sim 0.9$ seen in Figure 15 . Nevertheless, due to the large error uncertainties, all these results are poorly constrained.

The explosion fraction is around $0.0001-0.0003$ low$s$ SNe Ia per $M_{\odot}$ for all models except for the powerlaw fit to all corrected data $\left(t^{-2.7}\right)$ which is 0.001 low$s$ SNe Ia per $M_{\odot}$. These fractions are lower than for the normal- $s$ population -generally higher than $\sim 10^{-3}$ (e.g. Dahlen et al. 2004; Maoz et al. 2010b). Both populations differ by an order of magnitude which can be due either to a) a lower conversion efficiency $\eta$ of WD into SN Ia, or b) a lower progenitor mass range, taking into account that low mass stars are more frequent, or a combination of both. If one assumes that the conversion efficiency is constant over mass -this can be the case for certain considerations of the SD and DD scenarios (Pritchet et al. 2008) - the allowed mass range of low- $s$ SNe Ia would need to be extremely narrow. With a SalA IMF, and assuming that all main-sequence stars with $0.8 M_{\odot}<M<8 M_{\odot}$ become WDs, the cutoff value between low- $s$ and normal-s progenitors (from Eq. 7) would lie at $\sim 1 M_{\odot}$, an extremely narrow range of stars with too long delay-times $(>10 \mathrm{Gyr})$ to be seen at $z \gtrsim 0.3$. Changing the range of progenitors masses to $3 \widetilde{M}_{\odot}<M<8 M_{\odot}$ (Nomoto et al. 1994), the cutoff would need to be at $\sim 3.2 M_{\odot}$. On the other hand, using the lower WD mass limit of $1.95 M_{\odot}$ and cutoff at $2.10 M_{\odot}$, from the Gaussian distribution previously obtained, we also get a consistent explosion fraction ratio 


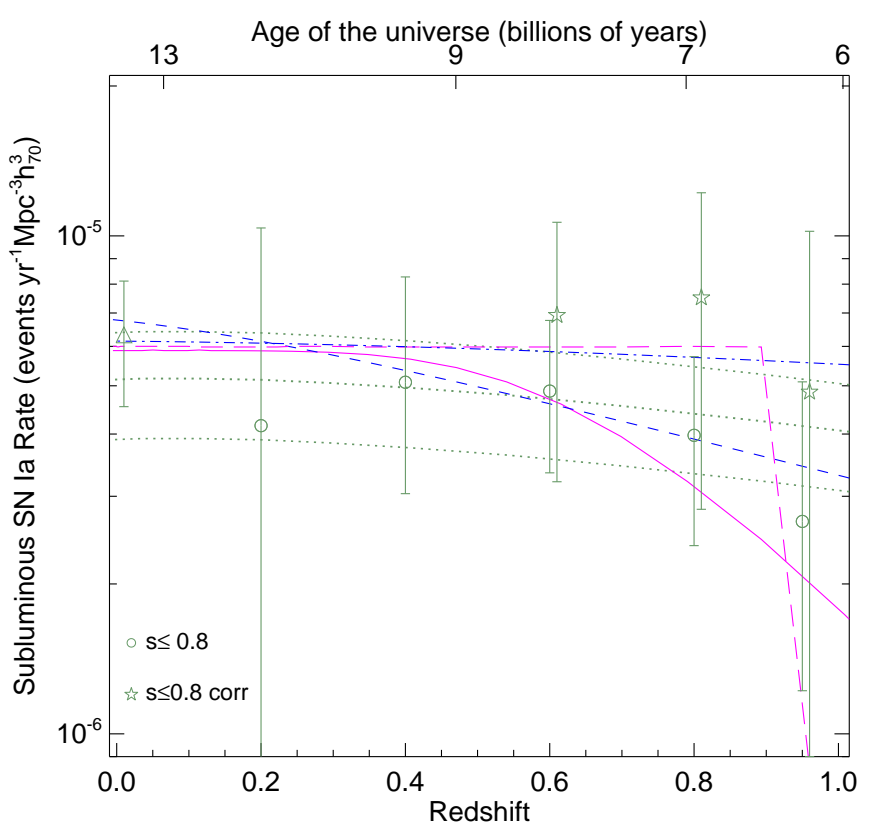

FIG. 15. - Best fit DTDs to the low- $s$ SN Ia rate. Up to $z<0.6$ : power-law (dashed blue) with $t^{-0.93 \pm 2.13}$, and Gaussian behaviour (solid purple) with $\tau=(5.11 \pm 5.65) \mathrm{Gyr}$ and $\sigma=0.94 \pm 3.16$. All corrected rates: power-law (dot-dashed blue) with $t^{-2.67 \pm 6.98}$ and Gaussian behaviour (long dashed purple) with $\tau=(5.88 \pm 4.84) \mathrm{Gyr}$ and $\sigma=0.15 \pm 2.41$. The dotted green lines represent the best $A$ model and errors for $z<0.6$ (see 5.3 )

of low- $s$ to normal- $s$.

\subsection{Extremely low-s SNe Ia}

We note that our sample does not consist of extremely subluminous SNe Ia. Looking at the local sample of typical subluminous objects, such as SN1991bg or SN1999by, the stretch is on the low end, i. e., $s=0.5-0.65$. In the SNLS $0.1<z<0.6$ range, we only obtain one such object (see Figure 16). This SN has a spectroscopic redshift of $z=0.57$ with LC parameters: $s=0.63 \pm 0.08$ and $c=0.02 \pm 0.06$. The candidate is hosted in a galaxy of mass $M=10^{10.47} M_{\odot}$ and sSFR $=10^{-11.93} M_{\odot} \mathrm{yr}^{-1}$, typical of very low-stretch objects.

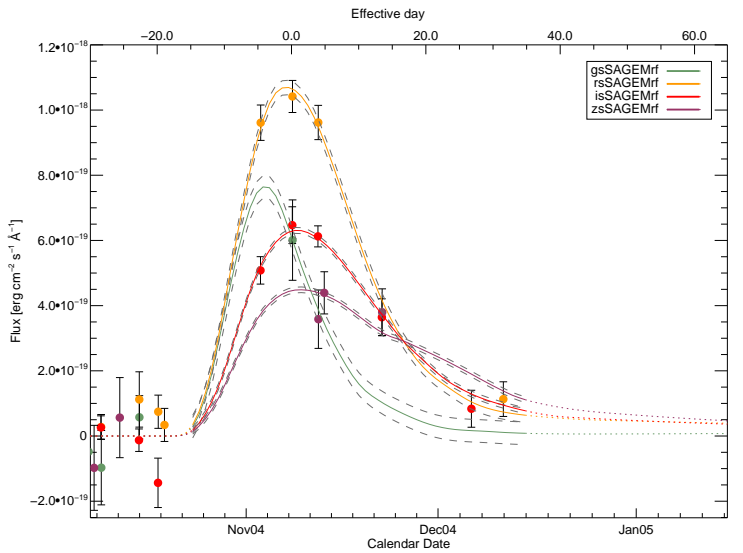

FIG. 16.- Extremely low- $s$ candidate, $04 \mathrm{D} 4 \mathrm{~ms}$, at spectroscopic redshift $z=0.57$ with $s=0.63 \pm 0.08$ and $c=0.02 \pm 0.06$.

This single SN corresponds to a single $z=0.35$ rate of $\left(0.25_{-0.21}^{+0.56}(\text { stat })_{-0.03}^{+0.06}(\mathrm{MC})\right) \times 10^{-6} \mathrm{yr}^{-1} \mathrm{Mpc}^{-3}$ (see Figure 177). Despite the obvious uncertainty in this number, it is tempting to argue that perhaps this "extremely low$s "$ population, i.e. $s<0.65$, actually behaves differently than our $s \leq 0.8$ group. If we maintain the extremely low- $s$ definition for the local luminosity-function sample of Li et al. (2010a) as well, we obtain 5 objects with $s \leq 0.65$ (5.16 corrected for completeness), which corresponds to $6.9_{-2.9}^{+4.5} \%$ of their rate (if we were to use the entire local sample, 15 objects have $s<0.65$, i.e., a $\sim 9 \%$ fraction), that is a rate of $(2.0 \pm 1.1) \times 10^{-6} \mathrm{yr}^{-1} \mathrm{Mpc}^{-3}$. We note that this is much smaller than the $91 \mathrm{bg}$ fractions generally quoted, such as the $17.9_{-6.2}^{+7.2} \%$ by Li et al. (2010a), indicating that this photometric definition is more restrictive than their definition (which is also based on spectra). Contrarily, if we were to use their fraction of "subluminous" SNe Ia to estimate the stretch limit, we would obtain $s \sim 0.75$. We emphasize therefore the importance of having a clear and consistent definition of "subluminous" SNe Ia across the redshift range. As shown in Figure 17 the rate evolution for this population also reveals a decline, perhaps even more rapid than for our standard low-s sample.

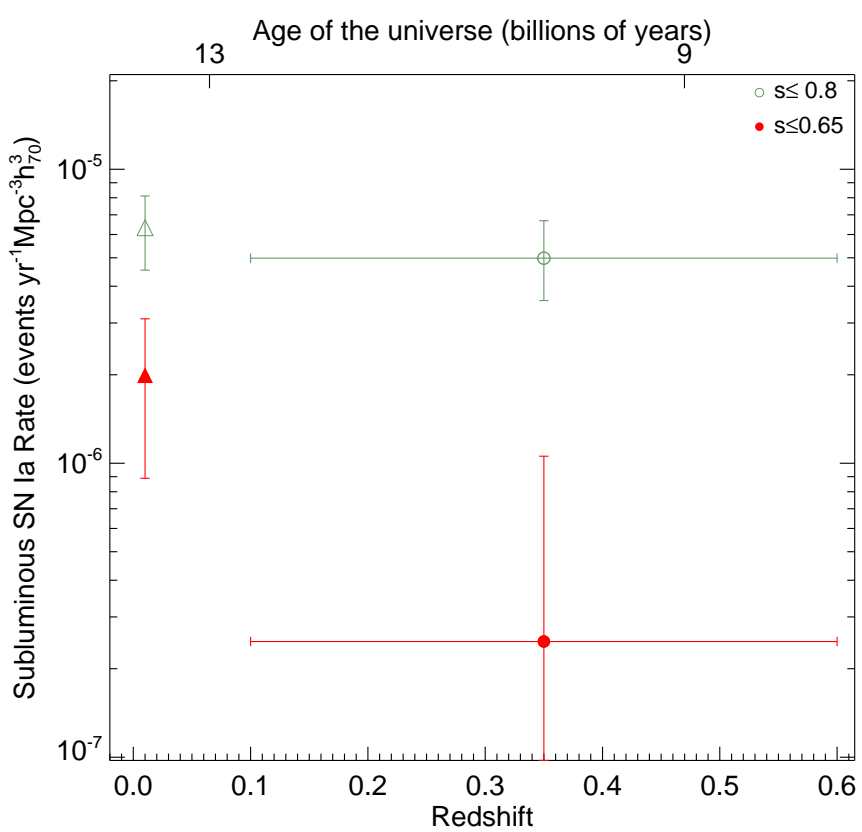

FIG. 17. - Single bin rate evolution for low- $s$ candidates $(s<$ $0.8)$ and extremely low- $s$ candidates with $s \leq 0.65$ from the SNLS (green and red circles respectively), and the estimated fraction of the SN Ia rate from Li et al. (2010b) (triangles). Horizontal error bars indicate the width of the z-bin.

\section{SUMMARY}

We have conducted a search in the SNLS for lowstretch SNe Ia, defined here as objects with $s \leq 0.8$. Typical "subluminous" surveys considered more extreme objects based also on spectroscopic features which have equivalent stretches of $\lesssim 0.72-0.75$. The definition chosen suits better our constructed color-stretch and magnitude-color-stretch relations trained with low- $z$ and SNLS objects. A lower transition value, nevertheless, provides also consistent LC relations. This argues for a continuous distribution of LC properties of SNe Ia, with low- $s$ SNe Ia having distinctive redder colors and a steeper color-stretch relation. The exact stretch value 
at which this transition occurs is not a sharp one and lies somewhere between and $0.69-0.87$.

The LC relations serve for the typing of low- $s$ SNe Ia at high redshift and for modeling the survey efficiency. We find 18 photometrically-identified low-stretch SNe Ia up to $z \sim 0.6$, indicating that the primary reason for their previous non-detections at high redshifts was due to selection effects. Most of the candidates at $z<0.6$ have $s>0.65$ and colors similar to the local sample. Only one of them is comparable to the faintest local counterparts such as $1991 \mathrm{bg}$. The candidates are observed to be primarily hosted in passive galaxies, with no star formation, as it is found locally. The spectroscopic candidates have no conclusive features due to low signal-to-noise.

We also find a population of blue low- $s$ SNe Ia at $z>0.6$ hosted mostly in quiet host envirnoments. They represent the blue end of the low- $s$ SN Ia sample at highredshift, whereas its reddest counterpart is not seen due to selection effects. A 30\% fraction of these have unusual blue colors that are mostly attributed to the larger photometric uncertainties at high- $z$. Nonetheless, some of them occur in star-forming galaxies and are possible CC-SN contaminants $(\lesssim 10 \%)$. Alternatively we speculate that they could represent a different set of objects that evolve rapidly but have bluer colors than typical low-s SNe.

We calculate a rate evolution of low- $s$ SNe Ia as a function of redshift and the results indicate a steady or slightly decreasing trend up to $z=0.6$. We correct the rates at $z>0.6$, where our sample is incomplete, and find the evolution to be different from the increase of the normal SN Ia population. The missclassification of CC-SNe as SNe Ia (or different unknown blue high- $z$ transients) is an important source of systematics in the rate measurements. This would overestimate the rate and should become larger with redshift, so that the effect of a decreasing rate would be even stronger. The main findings of this study are therefore not influenced by contamination.

An A-component model, proportional to just the mass of the galaxy, is sufficient to fit the rate. This is expected as most of the candidates are hosted in passive galaxies with no star formation. Low- $s$ SNe Ia are therefore a population coming from evolved and/or metalrich progenitors not dependent on the SFH. As shown in Howell et al. (2007), this has consequences for cosmological studies because the relative proportion of low to high stretch SNe Ia evolves at high redshift. The present study supports their findings at the extreme low-stretch end of the population. We find that $A+B$ models have an $A$-component with $\sim 30 \%$ coming from the low- $s$ population.

Studies of the DTD of different scenarios help constrain progenitors masses and models, as well as the conversion efficiency of WDs into SNe Ia. Despite the large uncertainties, the model of Strolger et al. (2010) fitted to our data hints to a Gaussian distribution centered around $\tau \simeq 5-6$ Gyr. The explosion fraction for those models is $\nu \sim 10^{-3}-10^{-4}$ low- $s$ SNe Ia per $M_{\odot}$, an order of magnitude lower than for normal- $s$ objects. These results again argue for the low- $s$ being part of a delayed component. Low- $s$ SNe Ia, together with other long delay SNe Ia of higher-s, could come from a separate progenitor channel. Although both, the SD and DD, scenarios can produce delayed SNe Ia, the merger of two low-mass WDs producing a sub-Chandrasekhar explosion seems more favorable to produce faint SNe in old stellar environments.

Finally, future surveys should be able to determine the rate evolution of low- $s$ much better and to higher redshifts where the systematic errors in this study become more significant. Spectroscopic redshifts of high- $z$ low$s$ SNe Ia are essential for this and could provide additional hint on their properties and their evolution. Further comparison of different SN Ia populations will give clues to understand the formation and explosion mechanisms.

We are grateful to the CFHT Queued Service Observations Team and the entire SNLS collaboration. This work has used observations obtained with MegaPrime/MegaCam, a joint project of CFHT and CEA/DAPNIA, at the Canada-France-Hawaii Telescope (CFHT) which is operated by the National Research Council (NRC) of Canada, the Institut National des Sciences de l'Univers of the Centre National de la Recherche Scientifique (CNRS) of France, and the University of Hawaii. This work is also based in part on data products produced at the Canadian Astronomy Data Centre as part of the CFHT Legacy Survey, a collaborative project of NRC and CNRS. We also use observations taken at the Gemini Observatory, which is operated by the Association of Universities for Research in Astronomy, Inc., under a cooperative agreement with the NSF on behalf of the Gemini partnership: the National Science Foundation (United States), the Science and Technology Facilities Council (United Kingdom), the National Research Council (Canada), CONICYT (Chile), the Australian Research Council (Australia), Ministério da Ciência e Tecnologia (Brazil) and Ministerio de Ciencia, Tecnología e Innovaci'on Productiva (Argentina). The Gemini program identification numbers are: GS-2003B-Q-8, GN-2003B-Q-9, GS-2004A-Q11, GN-2004A-Q-19, GS-2004B-Q-31,GN-2004B-Q-16, GS-2005A-Q-11, GN-2005A-Q-11, GS-2005B-Q-6, GN2005B-Q-7, GN-2006A-Q-7 and GN-2006B-Q-10. We acknowledge the support from our funding agencies: NSERC, CIAR, CNRS and CEA. MS acknowledges support from the Royal Society.

\section{REFERENCES}

Altavilla, G. et al. 2004, MNRAS, 349, 1344

Astier, P., Guy, J., Regnault, N., et al. 2006, A\&A, 447, 31

Balland, C. et al. 2009, A\&A, 507, 85

Bazin, G. et al. 2009, A\&A, 499, 653

Bildsten, L. et al. 2007, AJ, 662, L95
Brandt, T. D. et al. 2010, ArXiv:1002.0848

Bronder, T. J. et al. 2008, A\&A, 477, 717

Cappellaro, E., Evans, R., \& Turatto, M. 1999, A\&A, 351, 459

Conley, A. et al. 2008, ApJ, 681, 482

Dahlen, T. \& Fransson, C. 1999, A\&A, 350, 349 
Dahlen, T., Strolger, L.-G., \& Riess. 2008, AJ, 681, 462

Dahlen, T. et al. 2004, ApJ, 613, 189

Davis, M. et al. 2003, in Presented at the Society of

Photo-Optical Instrumentation Engineers (SPIE) Conference, Vol. 4834, Society of Photo-Optical Instrumentation Engineers (SPIE) Conference Series, ed. P. Guhathakurta, 161-172

Dilday, B. et al. 2008, AJ, 682, 262

—. 2010, ArXiv:1001.4995

Ellis, R. S. et al. 2008, ApJ, 674, 51

Filippenko, A. V. et al. 1992, AJ, 104, 1543

Foley, R. J. et al. 2003, PASP, 115, 1220

—. 2009, AJ, 137, 3731

Freeman, P. E. et al. 2009, MNRAS, 398, 2012

Gal-Yam, A., Ofek, E. O., \& Shemmer, O. 2002, MNRAS, 332, L73

Gamezo, V. N., Khokhlov, A. M., \& Oran, E. S. 2005, ApJ, 623, 337

Garnavich, P. M. et al. 2004, ApJ, 613, 1120

Gehrels, N. 1986, ApJ, 303, 336

Goldhaber, G. et al. 2001, ApJ, 558, 359

Guy, J. et al. 2007, A\&A, 466, 11

Guy, M. et al. 2010, submitted

Hamuy, M. et al. 1996, AJ, 112, 2391

-. 2000, AJ, 120, 1479

Hicken, M. et al. 2009, ApJ, 700, 331

Hoeflich, P. \& Khokhlov, A. 1996, ApJ, 457, 500

Hopkins, A. M. \& Beacom, J. F. 2006, ApJ, 651, 142

Howell, D. A. 2001, ApJ, 554, L193

Howell, D. A. et al. 2005, ApJ, 634, 1190

—. 2007, ApJ, 667, L37

Hoyle, F. \& Fowler, W. A. 1960, ApJ, 132, 565

Iben, I. \& Tutukov, A. V. 1984, ApJS, 54, 335

Isern, J., Canal, R., \& Labay, J. 1991, ApJ, 372, L83

James, F. \& Roos, M. 1975, Comput. Phys. Commun., 10, 343

Jha, S. et al. 2006, AJ, 131, 527

Jönsson, J. et al. 2010, in preparation

Kasen, D., Röpke, F. K., \& Woosley, S. E. 2009, Nature, 460, 869

Kasliwal, M. M. et al. 2008, ApJ, 683, L29

-. 2010, ApJ, 723, L98

Kowalski, M. et al. 2008, AJ, 686, 749

Krisciunas, K. et al. 2001, AJ, 122, 1616

Kuhlen, M., Woosley, S. E., \& Glatzmaier, G. A. 2006, ApJ, 640, 407

Le Borgne, D. \& Rocca-Volmerange, B. 2002, A\&A, 386, 446

Le Fèvre, O. et al. 2005, A\&A, 439, 845

Li, W. et al. 2001, ApJ, 546, 734

-. 2006, PASP, 118, 37

-. 2010a, ArXiv e-prints

—. 2010b, ArXiv e-prints

Lilly, S. J. et al. 2007, ApJS, 172, 70

Livne, E. 1990, ApJ, 354, L53

Livne, E., Asida, S. M., \& Höflich, P. 2005, ApJ, 632, 443

Mannucci, F. et al. 2005, A\&A, 433, 807
Maoz, D. \& Badenes, C. 2010, MNRAS, 968

Maoz, D., Sharon, K., \& Gal-Yam, A. 2010a, ArXiv e-prints

Maoz, D. et al. 2010b, ArXiv e-prints

Mazzali, P. A. et al. 2007, Science, 315, 825

Modjaz, M. et al. 2001, PASP, 113, 308

Neill, J. D. et al. 2006, AJ, 132, 1126

—. 2009, ApJ, 707, 1449

Nomoto, K. \& Iben, I. 1985, ApJ, 297, 531

Nomoto, K., Thielemann, F.-K., \& Yokoi, K. 1984, ApJ, 286, 644

Nomoto, K. et al. 1994, in Supernovae, ed. S. A. Bludman,

R. Mochkovitch, \& J. Zinn-Justin, 199-+

Pain, R. et al. 1996, ApJ, 473, 356

-. 2002, ApJ, 577, 120

Pakmor, R. et al. 2010, Nature, 463, 61

Pastorello, A. et al. 2004, MNRAS, 347, 74

-. 2008, MNRAS, 389, 113

Perets, H. B. et al. 2010, Nature, 465, 322

Perlmutter, S. et al. 1997, ApJ, 483, 565

Perrett, K. et al. 2010a, in preparation

-. 2010b, AJ, 140, 518

Phillips, M. M. et al. 1987, PASP, 99, 592

-. 2007, PASP, 119, 360

Pian, E. et al. 2006, Nature, 442, 1011

Poznanski, D. et al. 2010, Science, 327, 58

Pritchet, C. J., Howell, D. A., \& Sullivan, M. 2008, ApJ, 683, L25

Raskin, C. et al. 2009a, MNRAS, 399, L156

—. 2009b, ApJ, 707, 74

Riess, A. G. et al. 1999, AJ, 117, 707

Rosswog, S. et al. 2009, ApJ, 705, L128

Scannapieco, E. \& Bildsten, L. 2005, ApJ, 629, L85

Schawinski, K. 2009, MNRAS, 397, 717

Schlegel, D. J., Finkbeiner, D. P., \& Davis, M. 1998, ApJ, 500 525

Smartt, S. J. et al. 2009, MNRAS, 395, 1409

Sollerman, J. et al. 2006, A\&A, 454, 503

Strolger, L., Dahlen, T., \& Riess, A. G. 2010, ArXiv:1002.2494

Strolger, L.-G. et al. 2004, ApJ, 613, 200

Sullivan, M. et al. 2006a, AJ, 131, 960

-. 2006b, ApJ, 648, 868

Taubenberger, S. et al. 2008, MNRAS, 385, 75

Totani, T. et al. 2008, PASJ, 60, 1327

Tremonti, C. A. et al. 2004, ApJ, 613, 898

Tsvetkov, D. Y. 2006, Peremennye Zvezdy, 26

Turatto, M. et al. 1996, MNRAS, 283, 1

Tutukov, A. V. \& Yungelson, L. R. 1994, MNRAS, 268, 871

Valenti, S. et al. 2008, MNRAS, 383, 1485

Webbink, R. F. 1984, ApJ, 277, 355

Whelan, J. \& Iben, I. J. 1973, ApJ, 186, 1007

Woosley, S. E. \& Weaver, T. A. 1994, ApJ, 423, 371

Yoshii, Y. et al. 1988, AJ, 592, 467 
TABLE 1

NEARBY LOW- $s$ OBJECTS THAT PASSED THE OBSERVATION AND FIT CULLS USED IN THE ANALYSIS

\begin{tabular}{|c|c|c|c|}
\hline Name & Redshift & Stretch & Source \\
\hline SN1986g & 0.002 & 0.71 & Phillips et al. (1987) \\
\hline SN1990af & 0.050 & 0.75 & Hamuy et al. (1996) \\
\hline SN1991bg & 0.004 & 0.50 & Turatto et al. (1996),Filippenko et al. (1992) \\
\hline SN1992bl & 0.044 & 0.80 & Hamuv et al. (1996) \\
\hline SN1992bo & 0.019 & 0.79 & Hamuy et al. (1996) \\
\hline SN1992br & 0.088 & 0.60 & Hamuv et al. (1996) \\
\hline SN1993h & 0.024 & 0.66 & Hamuy et al. (1996), Altavilla et al. (2004) \\
\hline SN1994m & 0.023 & 0.76 & Riess et al. (1999), Altavilla et al. (2004) \\
\hline SN1995ak & 0.023 & 0.79 & Riess et al. (1999) \\
\hline SN1998bp & 0.010 & 0.63 & Jha et al. (2006) \\
\hline SN1998co & 0.018 & 0.65 & Jha et al. (2006) \\
\hline SN1998de & 0.017 & 0.64 & Modiaz et al. (2001) \\
\hline SN1999bm & 0.143 & 0.68 & Kowalski et al. (2008) \\
\hline SN1999by & 0.002 & 0.61 & Garnavich et al. (2004) \\
\hline SN1999da & 0.013 & 0.59 & Krisciunas et al. (2001) \\
\hline SN2000dk & 0.017 & 0.77 & Jha et al. (2006) \\
\hline SN2001da & 0.017 & 0.61 & Hicken et al. (2009) \\
\hline SN2002cx & 0.024 & 0.57 & Phillips et al. (2007) \\
\hline SN2002hw & 0.018 & 0.73 & Hicken et al. (2009) \\
\hline SN2003ic & 0.056 & 0.77 & Hicken et al. (2009) \\
\hline SN2003iv & 0.034 & 0.74 & Hicken et al. (2009) \\
\hline SN2005am & 0.008 & 0.71 & Hicken et al. (2009), Li et al. (2006) \\
\hline SN2005bl & 0.024 & 0.60 & Taubenberger et al. (2008) \\
\hline SN2005hf & 0.043 & 0.76 & Hicken et al. (2009) \\
\hline SN2005ke & 0.005 & 0.69 & Hicken et al. (2009) \\
\hline SN2005mc & 0.025 & 0.69 & Hicken et al. (2009) \\
\hline SN2006ak & 0.038 & 0.78 & Hicken et al. (2009) \\
\hline SN2006al & 0.068 & 0.78 & Hicken et al. (2009) \\
\hline SN2006br & 0.025 & 0.78 & Hicken et al. (2009) \\
\hline SN2006bw & 0.030 & 0.71 & Hicken et al. (2009) \\
\hline SN2006bz & 0.028 & 0.53 & Hicken et al. (2009) \\
\hline SN2006gj & 0.028 & 0.71 & Hicken et al. (2009) \\
\hline SN2006gt & 0.045 & 0.53 & Hicken et al. (2009) \\
\hline SN2006hb & 0.015 & 0.69 & Hicken et al. (2009) \\
\hline SN2006je & 0.038 & 0.48 & Hicken et al. (2009) \\
\hline SN2006mo & 0.037 & 0.73 & Hicken et al. (2009) \\
\hline SN2006n & 0.014 & 0.77 & Hicken et al. (2009) \\
\hline SN2006nz & 0.038 & 0.62 & Hicken et al. (2009) \\
\hline SN2006ob & 0.059 & 0.75 & Hicken et al. (2009) \\
\hline SN2006td & 0.016 & 0.79 & Hicken et al. (2009) \\
\hline SN2007al & 0.012 & 0.47 & Hicken et al. (2009) \\
\hline SN2007au & 0.021 & 0.67 & Hicken et al. (2009) \\
\hline SN2007ax & 0.007 & 0.53 & Hicken et al. (2009) \\
\hline SN2007ci & 0.018 & 0.79 & Hicken et al. (2009) \\
\hline
\end{tabular}

TABLE 2

Nearby non-Ia SNe with acceptable SN Ia SiFtO fits, enough data coverage and fitted $s \leq 0.8$.

\begin{tabular}{|c|c|c|c|c|}
\hline \hline Name & Type & Redshift & Stretch & Source \\
\hline SN1999eu & SNIIP & 0.004 & 0.45 & Pastorello et al. (2004) \\
SN2001b & SNIb & 0.005 & 0.60 & Tsvetkov (2006) \\
SN2002ao & SNIbn & 0.005 & 0.54 & Pastorello et al. (2008) \\
SN2002ap & SNIc & 0.002 & 0.68 & Gal-Yam et al. (2002); Foley et al. (2003); Yoshii et al. (1988) \\
SN2003jd & SNIc & 0.019 & 0.73 & Valenti et al. (2008) \\
SN2006aj & SNIc & 0.033 & 0.73 & Pian et al. (2006); Sollerman et al. (2006) \\
\hline
\end{tabular}


TABLE 3

Culls APPlied TO THE training SAMPle

\begin{tabular}{|c|c|c|c|c|}
\hline Cull & \multicolumn{3}{|c|}{ Number } \\
& \multicolumn{2}{|c|}{ confirmed } & \multicolumn{2}{c|}{ confirmed } \\
& SNe Ia(low- $s$ ) & \multicolumn{2}{c|}{ CC-SNe(low- $s$ ) } \\
& low- $z$ & SNLS & low- $z$ & SNLS \\
\hline None & $246(57)$ & $112(3)$ & $37(12)$ & $47(9)$ \\
observation & $207(44)$ & $106(3)$ & $14(6)$ & $21(4)$ \\
$\chi_{\text {SiFTO }}^{2}$ and snakes & $184(41)$ & $97(3)$ & $2(2)$ & $1(0)$ \\
\hline
\end{tabular}

TABLE 4

Culls applied to the SNLS SAmple

\begin{tabular}{|c|c|c|c|c|}
\hline Cull & \multirow{3}{|c|}{ Number } \\
& all & low-s & $\begin{array}{c}\text { confirmed } \\
\text { SNe Ia }\end{array}$ & $\begin{array}{c}\text { confirmed } \\
\text { CC-SNe }\end{array}$ \\
\hline None & 5234 & 1497 & 407 & 86 \\
observation & 2615 & 837 & 343 & 62 \\
$\chi_{\text {SiFTO }}^{2}$ & 1054 & 144 & 338 & 22 \\
$\chi_{\text {estimate_sn }}^{2}$ & 925 & 118 & 329 & 8 \\
$\chi_{f, \text { estimate_sn }}^{2}$ & 848 & 109 & 303 & 5 \\
snakes & 710 & 70 & 295 & 1 \\
\hline FINAL & 710 & 70 & 295 & 1 \\
$z<0.6$ & 174 & 18 & 118 & 0 \\
\hline
\end{tabular}

TABLE 5

VOlumetric RATES FOR THE SUbluminous SNe IA IN THE SNLS

First AND SECOND COLUMN SHOW THE REDSHIFT AT THE MIDDLE OF THE BIN AND THE MEAN REDSHIFT RESPECTIVELY. The THIRD COLUMN IS THE NUMBER OF OBJECTS PER $z$-BIN AND THE FOURTH IS THE MEAN WEIGHT $\left(w_{i}=\left(1+z_{i}\right) /\left(\epsilon_{i} \times \Delta T_{i}\right)\right.$, WHERE $\epsilon_{i}$ IS THE EFFICIENCY) used for the Poisson errors. Fifth COlumn indicate the measured rate With Statistical Weighted Poisson errors, and SiXth column the Monte-Carlo rates with weighted Poisson, Monte-Carlo and contamination errors. The seventh column shows THE CORRECTION FACTOR (WITH ERRORS) APPLIED TO GET THE LAST COLUMN HIGH- $z$ RATES CORRECTED FOR THE COLOR DISTRIBUTION AT $z<0.6$.

\begin{tabular}{|c|c|c|c|c|c|c|c|}
\hline \hline$z_{\text {mid }}$ & $\langle z\rangle$ & $N$ & $\langle w\rangle, \sigma_{w}$ & $\begin{array}{c}r_{\text {meas }} \\
\left(10^{-5} \mathrm{yr}^{-1} \mathrm{Mpc}^{-3} h_{70}^{3}\right)\end{array}$ & $\begin{array}{c}r_{\mathrm{MC}} \\
\left(10^{-5} \mathrm{yr}^{-1} \mathrm{Mpc}^{-3} h_{70}^{3}\right)\end{array}$ & $\begin{array}{c}f_{\text {corr }} \\
\left(10^{-5} \mathrm{yr}^{-1} \mathrm{Mpc}^{-3} h_{70}^{3}\right)\end{array}$ \\
\hline 0.20 & 0.17 & 2 & $1.40,0.75$ & $0.46_{-0.31}^{+0.68}$ & $0.42_{-0.28}^{+0.61}(\mathrm{stat})_{-0.17}^{+0.01}(\mathrm{MC}) \pm 0.08(\mathrm{cont})$ & - & - \\
0.40 & 0.43 & 9 & $1.08,0.24$ & $0.74_{-0.28}^{+0.41}$ & $0.51_{-0.21}^{+0.30}(\text { stat })_{-0.08}^{+0.16}(\mathrm{MC}) \pm 0.11(\mathrm{cont})$ & - & - \\
0.60 & 0.63 & 19 & $1.16,0.21$ & $0.65_{-0.15}^{+0.19}$ & $0.49_{-0.16}^{+0.20}(\text { stat })_{-0.10}^{+0.12}(\mathrm{MC}) \pm 0.13(\mathrm{cont})$ & $1.42 \pm 0.20$ & $0.69_{-0.29}^{+0.35}$ \\
0.80 & 0.78 & 18 & $1.44,0.65$ & $0.44_{-0.11}^{+0.15}$ & $0.40_{-0.19}^{+0.24}(\mathrm{stat})_{-0.18}^{+0.11}(\mathrm{MC}) \pm 0.12(\mathrm{cont})$ & $1.89 \pm 0.26$ & $0.75_{-0.43}^{+0.51}$ \\
0.95 & 0.94 & 11 & $2.37,3.02$ & $0.48_{-0.20}^{+0.30}$ & $0.27_{-0.19}^{+0.29}(\mathrm{stat})_{-0.18}^{+0.18}(\mathrm{MC}) \pm 0.08(\mathrm{cont})$ & $1.82 \pm 0.40$ & $0.49_{-0.40}^{+0.62}$ \\
\hline \hline
\end{tabular}

